\title{
The Application of S-Band Polarimetric Radar Measurements to Ka-Band Attenuation Prediction
}

\author{
JOHN D. BEAVER AND V. N. BRINGI
}

\begin{abstract}
In September 1993, the National Aeronautics and Space Administration's Advanced Communications Technology Satellite (ACTS) was deployed into a geostationary orbit near $100^{\circ} \mathrm{W}$ longitude. The ACTS satellite employs two Ka-band beacons, one at $20.185 \mathrm{GHz}$ and another at $27.505 \mathrm{GHz}$. Impairments due to rain attenuation and tropospheric scintillations will significantly affect new technologies for this spectrum. Heavy rain at Ka-band can easily produce $30 \mathrm{~dB}$ of attenuation along the propagation path. Propagation experiments being conducted in seven different climatic zones involve multiyear attenuation measurements along the satellite-earth slant path. Measurements in the B2 climatic zone are made with an ACTS propagation terminal located in northeast Colorado. In order to gain more understanding about the physical processes that are responsible for Ka-band attenuation, the Colorado State University CHILL $S$-band polarimetric radar is used to take radar measurements along the slant path. The Colorado Front Range experiences a variety of weather conditions throughout the year ranging from upslope rain conditions to winter storms. Four such events measured along the slant path are illustrated in this paper. They include two convective cases and two "bright-band" cases. The $S$-band polarimetric radar data is used to initialize radar-based attenuation-prediction models, which are applied to the four precipitation events described. The comparisons of predicted attenuation to measured attenuation are quite good. It was also found during the course of the experiment that water droplets standing on the antenna surface can cause appreciable attenuation at Ka-band frequencies. That finding needs to be recognized in future model development and statistical analysis.
\end{abstract}

Keywords-ACTS, attenuation measurement, meteorological radar, polarimetry, prediction methods, propagation, satellite communication.

\section{INTRODUCTION}

With an increased demand in satellite communications, the current spectrum allocations at $\mathrm{C}$ - and $\mathrm{Ku}$-bands are quickly becoming overcrowded. This has led to an increased interest from industry to utilize the Ka-band fre-

Manuscript received August 20, 1996; revised March 3, 1997. This work was supported by NASA under Contract \#NAS3-26410. The CSUCHILL radar is funded by a cooperative agreement with the National Science Foundation under ATM-9500 108.

The authors are with Colorado State University, Department of Electrical Engineering, Fort Collins, CO 80523-1373 USA.

Publisher Item Identifier S 0018-9219(97)04656-2. quency spectrum. Currently, several industry leaders are in the process of developing satellite communications systems that operate at the Ka-band frequencies or already have systems in place. Benefits of operating at the Ka-band frequencies include increases in data transmission rates and the amount of data transmitted, smaller earth receiving stations, which leads to greater mobility, and (for the time being) the use of a portion of the spectrum that is sparsely used. Along with the benefits of operating at these frequencies, however, there are some disadvantages, such as increased attenuation effects due to atmospheric conditions.

One of the attractive features that led to the use of C-and $\mathrm{X}$-bands for satellite communications was the low susceptibility to attenuation effects caused by rain or clouds. The longer wavelengths are minimally affected by atmospheric conditions. The Ka-band frequencies, however, are very susceptible to weather-related events. Rain, clouds, and even gaseous absorption by oxygen and water vapor can adversely affect the signal and must be considered. Rain can easily produce $20-30 \mathrm{~dB}$ of attenuation at the Ka-band frequencies. Therefore, before widespread commercial use, propagation effects at Ka-band must be studied.

To help further the study of propagation effects at Kaband frequencies, an experimental communications satellite using Ka-band technology was developed by the National Aeronautics and Space Administration (NASA). In September 1993, the Advanced Communications Technology Satellite (ACTS) was deployed into a geostationary orbit near $100^{\circ} \mathrm{W}$ longitude by the space shuttle Discovery. The ACTS system supports both communication and propagation experiments at the $20 / 30 \mathrm{GHz}$ frequency bands. The propagation experiment involves multiyear attenuation measurements along the satellite-earth slant path. The ACTS campaign is designed to build on previous studies conducted at Ka-band frequencies, including those conducted in the United States with the COMSTAR satellite [1], [2] as well as the Japanese and European ventures such as BSE, CS, ATS-6, SIRIO, OTS, and most recently the OLYMPUS campaign [3]. 
Colorado State University (CSU) and six other sites across the United States and Canada are conducting the propagation studies. Each site is equipped with the ACTS propagation terminal (APT). The APT's were designed and built by Virginia Polytechnic Institute and State University's Satellite Communications Group [4] and are receiveonly earth stations. Each site is located in a different climatic zone, with CSU in the newly designated B2 climate zone as defined by the Crane-Global rain model [5]. In addition to the Colorado site, other propagation sites include British Columbia, Alaska, New Mexico, Oklahoma, Florida, and Maryland.

The main thrust for the propagation experiment is to obtain high quality attenuation measurements in order to construct a data base so that the attenuation effects at Ka-band frequencies can be statistically characterized on a monthly and annual basis. In addition to the main goal, each site is applying its own expertise to secondary studies. CSU's contribution is the application of polarimetric radar data for attenuation prediction. Radar data taken by the CSU CHILL fully polarimetric, Doppler radar is used to gain a greater understanding of the microphysical processes that are responsible for Ka-band attenuation that occurs along the ACTS slant path. Radar data are also used to initialize a radar-based attenuation-prediction model based on reflectivity and specific differential phase.

Polarimetric radars have been used previously in studying propagation effects along earth-satellite paths. For example, Antar et al. [6] used a $16.5-\mathrm{GHz}$ circularly polarized radar to study differential attenuation and differential phase shift due to rain and ice along the propagation path, with similar measurements made with the COMSTAR beacon. The application of dual linearly polarized radar, in particular the use of differential reflectivity $\left(Z_{\mathrm{DR}}\right)$ in predicting slant-path rain attenuation along earth-satellite paths, was initially due to Cherry et al. [7]. Goddard and Cherry [8] report on an attenuation-prediction model using S-band radar measurements of reflectivity and $Z_{\mathrm{DR}}$ along the slant path and found excellent agreement with directly measured 11.6-GHz beacon attenuation. A similar early application of S-band dual-polarized radar using $Z_{\mathrm{DR}}$ measurements to predict $11.6-\mathrm{GHz}$ attenuation (SIRIO satellite beacon) due to rain is described in [9]. More recently, Goddard et al. [10] described the new capabilities of the Chilbolton radar, which include the measurement of differential phase and linear depolarization ratio to attenuation prediction at 12.5, 20, and $30 \mathrm{GHz}$, the three beacon frequencies on the OLYMPUS satellite. The OLYMPUS Propagation Experiment involved two S-band and two C-band dual linearly polarized radars, all located in Europe, studying propagation effects due to rain, melting layer, and ice along the slant path in coordination with OLYMPUS beacon measurements [11]. Thus, the use of the fully polarimetric CSU-CHILL radar in attenuation prediction for the ACTS propagation experiments continues along the tradition initialized by the Chilbolton radar group at the Rutherford Appleton Laboratory in the United Kingdom [10], as well as the other OLYMPUS dual-polarized radar experimenters.
This paper presents an overview and the results of using CSU-CHILL S-band radar data to further the understanding of Ka-band attenuation. Section II presents a brief background of the CSU-CHILL radar facility and definitions of several polarimetric radar parameters. The development of the radar-based attenuation models is also presented in Section II. Section III presents four case studies that have been obtained during the course of the experiment. CSUAPT attenuation data are presented along with concurrent CSU-CHILL radar data for each precipitation event. Application of the radar-based attenuation models for each case study is presented in Section IV, along with a discussion of the results. Last, Section V presents conclusions that were obtained from the experiment.

\section{OVERVIEW AND MODEL DEVElopMENT}

\section{A. CSU-CHILL Radar Description}

The CSU-CHILL radar was originally designed and constructed jointly by the University of Chicago and the Illinois State Water Survey [12]. In 1990, the CHILL radar was moved to its present location outside of Greeley, $\mathrm{CO}$, and is now used exclusively as a research radar operated by CSU under the sponsorship of the National Science Foundation. It was recently upgraded to a fully polarimetric S-band radar that can alternately transmit two orthogonally polarized signals and simultaneously receive the co- and cross-polarized signals. A summary of the system characteristics is given in Table 1. A new dual-polarized antenna with excellent sidelobes (worst case $-27 \mathrm{~dB}$ in any $\phi$-plane) and polarization purity (linear depolarization ratio system limit of -32 to $-34 \mathrm{~dB}$ ) was installed in early 1995 . The use of two separate transmitters eliminates the need for a high power polarization switch, ensuring a high system isolation $(>50 \mathrm{~dB})$ between channels.

\section{B. Radar Observables}

The CSU-CHILL radar transmits and receives both horizontal and vertical polarizations. The ability to measure both the copolar and cross-polar returns allows measurements of such quantities as the horizontal reflectivity $\left(Z_{\mathrm{HH}}\right)$, differential reflectivity $\left(Z_{\mathrm{DR}}\right)$, differential phase between the $\mathrm{H}$ and $\mathrm{V}$ copolar signals $\left(\Phi_{\mathrm{DP}}\right)$, correlation coefficient between the two copolar signals $\left(\rho_{\mathrm{HV}}\right)$, and linear depolarization ratio (LDR). An excellent explanation of these parameters and their application to precipitation measurements can be found in [13].

The radar observables can be defined in terms of the forward- and backscattering amplitudes and the raindrop size distribution. The first subscript of the polarization states given in the following equations refers to the received polarization state, while the second subscript refers to the transmitted polarization state. The horizontal and vertical reflectivities are defined by

$Z_{\mathrm{HH}, \mathrm{VV}}=\frac{\lambda^{4}}{\pi^{\tilde{5}}|K|^{2}} \int \sigma_{\mathrm{HH}, \mathrm{VV}}(D) N(D) d D \quad\left(\mathrm{~mm}^{6} \mathrm{~m}^{-3}\right)$ 
Table 1 System Characteristics of the CSU-CHILL Radar

\begin{tabular}{|c|c|}
\hline \multicolumn{2}{|r|}{ Antenna } \\
\hline type: & fully steerable, prime focus parabolic reflector \\
\hline size: & $8.5 \mathrm{~m}$ \\
\hline feed: & scalar horn \\
\hline 3 dB beamwidth: & $1.0^{\circ}$ \\
\hline directivity : & $45 \mathrm{~dB}$ \\
\hline sidelobe level (any $\phi$-plane): & $\leq-27 \mathrm{~dB}$ \\
\hline cross-pol. level (any $\phi$-plane): & $\leq-30 \mathrm{~dB}$ \\
\hline polarization radiated: & Horizontal or Vertical \\
\hline \multicolumn{2}{|r|}{ Transmitter } \\
\hline type: & klystron, modernized FPS-18 \\
\hline wavelength: & $10.7 \mathrm{~cm}$ \\
\hline peak Power: & $700-1000 \mathrm{~kW}$ \\
\hline pulse width: & steps of $0.1 \mu \mathrm{s}$ up to a max. of $1 \mu \mathrm{s}$ \\
\hline PRT: & $800 \cdots 2500 \mu \mathrm{s}$ \\
\hline max. unambigu. range: & $375 \mathrm{~km}$ \\
\hline max. unambigu. $v$ & $\pm 34.3 \mathrm{~m} / \mathrm{s}$ \\
\hline \multicolumn{2}{|r|}{ Receiver } \\
\hline noise figure: & $0.7 \mathrm{~dB}$ \\
\hline noise power: & $-114 \mathrm{dBm}$ \\
\hline typical bandwidth: & $750 \mathrm{kHz}$ \\
\hline transfer function: & linear \\
\hline dynamic range: & $90 \mathrm{~dB}, 0-60 \mathrm{~dB}$ IAGC in $12 \mathrm{~dB}$ steps \\
\hline \multicolumn{2}{|r|}{ Data Acquisition } \\
\hline signal processor: & SP20 made by Lassen Research \\
\hline number of range gates: & $64-2048$ \\
\hline range gate spacing: & $0.2 \mu \mathrm{s}$ or $1 \mu \mathrm{s}$ \\
\hline sampling rate/avg. option: & under micro-code control \\
\hline video digitizer: & 12-bit, in the SP20 input card for $I, Q$ and $\log P$ \\
\hline time series capability: & up to 150 range gates. \\
\hline \multicolumn{2}{|r|}{ Variables Available } \\
\hline \multicolumn{2}{|c|}{$\begin{array}{l}\text { - Reflectivity at H polarization }\left(Z_{h}\right) \\
\text { - Differential Reflectivity }\left(Z_{d r}\right) \\
\text { - Mean Doppler Velocity }(\bar{v}) \text { and Spectral Width }\left(\sigma_{v}\right) \\
\text { - Differential Phase between H and V states }\left(\Psi_{d p}\right) \\
\text { - Copolar Correlation Coefficient }\left(\rho_{h v}(0)\right) \\
\text { - Linear Depolarization Ratio (LDR) } \\
\text { - Doppler Spectra from FFT processing } \\
\text { - I, Q and logP for every pulse in timc scries mode (up to } 150 \text { gates) }\end{array}$} \\
\hline
\end{tabular}

where $\sigma_{\mathrm{HH}, \mathrm{vV}}(D)$ are the copolar radar cross sections at the horizontal and vertical polarizations, $|K|=\left(\epsilon_{r}-\right.$ 1)/ $\left(\epsilon_{r}+2\right), \epsilon_{r}$ is the dielectric constant of water, and $\lambda$ is the wavelength. Differential reflectivity is defined as

$$
Z_{\mathrm{DR}}=10 \log \frac{Z_{\mathrm{HH}}}{Z_{\mathrm{VV}}} \quad(\mathrm{dB}) .
$$

Defining $f_{\mathrm{HH}}$ and $f_{\mathrm{VV}}$ as the forward-scattering amplitudes of the $\mathrm{H}$ and $\mathrm{V}$ polarized waves, respectively, the specific differential phase is given as

$$
\begin{array}{r}
K_{\mathrm{DP}}=\frac{180 \lambda}{\pi} \int \operatorname{Re}\left[f_{\mathrm{HH}}(D)-f_{\mathrm{VV}}(D)\right] N(D) d D \\
\left({ }^{\circ} \mathrm{km}^{-1}\right) .
\end{array}
$$

Then $\Phi_{\mathrm{DP}}$ is defined as

$$
\Phi_{\mathrm{DP}}=\int_{r 1}^{r 2} 2 K_{\mathrm{DP}}(r) d r \quad(\mathrm{deg})
$$

where $\Phi_{\mathrm{DP}}$ is the two-way differential phase between range locations $r_{1}$ and $r_{2}$. If the backscatter amplitudes for the horizontal and vertical polarizations are defined as $S_{\mathrm{HH}}$ and $S_{\mathrm{VV}}$, then the copolar-correlation coefficient is given as

$$
\rho_{\mathrm{HV}}=\frac{\int S_{\mathrm{HH}}^{*}(D) S_{\mathrm{VV}}(D) N(D) d D}{\left\{\left[\int\left|S_{\mathrm{HH}}\right|^{2} N(D) d D\right]\left[\int\left|S_{\mathrm{VV}}\right|^{2} N(D) d D\right]\right\}^{1 / 2}} .
$$

The linear depolarization ratio can also be defined in terms of the backscatter amplitudes

$$
\mathrm{LDR}_{\mathrm{VH}}=10 \log \frac{\left|S_{\mathrm{VH}}\right|^{2}}{\left|S_{\mathrm{HH}}\right|^{2}} \quad(\mathrm{~dB}) .
$$

$N(D)$ is the raindrop size distribution and is the number of raindrops per unit volume per unit size interval $D$ 
to $D+\delta D$, where $D$ refers to the equivalent spherical diameter.

Each polarimetric parameter provides information about the type, shape, and orientation of particles present in a given radar range resolution volume [14]. For instance, information regarding the reflectivity-weighted mean axis ratio of oblate raindrops can be obtained from the differential reflectivity, while the linear depolarization ratio is sensitive to the mean hydrometeor shape and orientation away from the principal H/V directions. Specific differential phase is a good measure of rainfall intensity. While each of these parameters provides information on its own, looking at a combination of polarimetric parameters can give an even greater insight as to what is occurring in a particular storm cell [13]. A good example is the combination of horizontal reflectivity and differential reflectivity. Typically, if hail is present in a range resolution volume, the horizontal reflectivities tend to be high, while the tumbling nature of hail stones results in low values for differential reflectivity. This is just one of many examples of how polarimetric parameters can be used in describing hydrometeor types.

\section{Prediction of Ka-Band Attenuation from Radar Parameters}

The prediction of attenuation along an earth-satellite slant path using radar reflectivity measurements at a single polarization and at a nonattenuating frequency has been the subject of research since at least the early 1970's [2]. A simple power-law equation of the form $k=a Z_{\mathrm{HH}}^{b}$ where $k$ is the specific attenuation (or attenuation coefficient $\mathrm{dB} \mathrm{km}^{-1}$ ) and $Z_{\mathrm{HH}}$ is the radar reflectivity at horizontal polarization $\left(\mathrm{mm}^{6} \mathrm{~m}^{-3}\right)$ has been commonly used. Typically, the coefficients $a, b$ are based on measured raindrop size distributions at the ground or on assumed raindrop size distributions of the exponential or gamma form. The radar must be accurately calibrated to within $\pm 1 \mathrm{~dB}$ to achieve good comparisons between radar-predicted and directly measured path attenuation. The early experiments reviewed by Goldhirsh [2] adjusted the radar constant (which relates the backscatter power to $Z_{\mathrm{HH}}$ ) by 3-9 $\mathrm{dB}$ to force agreement between the radar-predicted and directly measured path attenuation. These adjustments were made relative to the original independently derived radar constants for each radar system. In all five cases reviewed, $Z_{\mathrm{HH}}$ had to be increased by 3-9 dB in spite of different radars' being used under different experimental conditions. In this paper, we do not use the ACTS attenuation data to adjust the radar constant. Rather, we independently adjust the radar constant from its nominal value in a manner discussed later using dual-polarized data, and any systematic discrepancy between the ACTS attenuation and radar-predicted attenuation (via $k=a Z_{\mathrm{HH}}^{b}$ ) is explained in Section IV.

In addition to the variability in the $k-Z_{\mathrm{HH}}$ relation due to raindrop size distribution fluctuations, it is important to consider the effects of wet ice hydrometeors along the propagation path. In particular, modeling the radar "bright band" due to melting snow has been an active research effort within the OLYMPUS propagation community [11]. In practice, an average $k-Z_{\mathrm{HH}}$ relation (based on brightband models) is used since the models require, among other parameters, the initial mass density of the snow particles, which is usually unavailable. The models also ignore physically important processes like ice crystal aggregation and breakup and, thus, are of limited applicability. In this paper, therefore, we do not use any bright-band model results.

With the advent of dual-polarized radars, the specific attenuation is related to both $Z_{\mathrm{HH}}$ and $Z_{\mathrm{DR}}$ in an attempt to account for the drop size distribution (DSD) variations [8]. Raindrops at terminal fall speed acquire an oblate spheroidal shape in response to a balance between surface tension, gravitational forces, and aerodynamic forces [15]. Small drops (diameter $\leq 1 \mathrm{~mm}$ ) are nearly spherical, while the larger drops are more oblate. In the Rayleigh scattering limit, $Z_{\mathrm{DR}}$ estimates the reflectivity-weighted mean axis ratio of the raindrops [16], and because of the nearly linear relation between axis ratio and (volume equivalent spherical) diameter, it also is a very good estimator of the reflectivity-weighted mean diameter of the raindrop distribution. The $k-\left(Z_{\mathrm{HH}}, Z_{\mathrm{DR}}\right)$ relation is generally based on DSD's of the form $N(D)=N_{0} e^{-\Lambda D}$ or $N(D)=N_{0} D^{2} e^{-\Lambda D}$ with $Z_{\mathrm{DR}}$ estimating the parameter $\Lambda$ and $N_{0}$ being determined by $Z_{\mathrm{HH}}$ once $\Lambda$ is fixed [8]. The accuracy of attenuation prediction depends on accurate calibration of both $Z_{\mathrm{HH}}$ (to within $\pm 1 \mathrm{~dB}$ or better) and $Z_{\mathrm{DR}}$ (to within $\pm 0.1 \mathrm{~dB}$ or better), which is achievable with research radars. Errors due to $N(D)$ 's deviating from the assumed forms (or raindrop oscillations, which cause slight departures from equilibrium shapes) or the presence of wet ice hydrometeors are difficult to account for. Nevertheless, the method has been applied with good success by the Rutherford Appleton group [10] to predict slant-path attenuation at $12.5,20$, and $30 \mathrm{GHz}$.

Recently, the specific differential phase $\left(K_{\mathrm{DP}}\right)$ has been used with excellent success to estimate rain rate $(R)$ with several practical advantages (see chapter 8 of [13] and references therein). Because $K_{\mathrm{DP}}$ is derived from radar measured $\Phi_{\mathrm{DP}}$ (the differential propagation phase), it is independent of the absolute system gain (or radar constant). It is also relatively insensitive to ice hydrometeors (such as hail) if it is assumed that the particles are "quasi" spherical, giving $f_{\mathrm{HH}} \approx f_{\mathrm{VV}}$ in (3). Simulations have shown that the $K_{\mathrm{DP}}-R$ relation is relatively insensitive to raindrop size distribution fluctuations [17]. At $\mathrm{S}$-band, the $K_{\mathrm{DP}}-R$ relation is in the form $K_{\mathrm{DP}}=40.5(R)^{0.845}$ where $K_{\mathrm{DP}}$ is in units of ${ }^{\circ} \mathrm{km}^{-1}$ while $R$ is in units of $\mathrm{mmh}^{-1}$ [17]. Because Ka-band specific attenuation is known to be nearly linearly related to $R$, it is logical to relate $k$ directly to $K_{\mathrm{DP}}$, and this is one of the methods used in this paper for attenuation prediction. Because the measurement accuracy of $K_{\mathrm{DP}}$ is around $\pm 0.3{ }^{\circ} \mathrm{km}^{-1}$, it is useful only at higher rain rates $\left(R \geq 20 \mathrm{mmh}^{-1}\right)$.

Another practical advantage of $K_{\mathrm{DP}}$ is that it can be used to adjust the nominal radar constant in a self-consistent manner using the rain medium itself [17]. Radar measurements of $Z_{\mathrm{HH}}, Z_{\mathrm{DR}}$, and $K_{\mathrm{DP}}$ are obtained in cases of 
known rain events, and a scatter plot of rain rate derived from $K_{\mathrm{DP}}$ versus rain rate derived from $\left(Z_{\mathrm{HH}}\right.$ and $\left.Z_{\mathrm{DR}}\right)$ is constructed. Because $K_{\mathrm{DP}}$ does not depend on absolute gain and $Z_{\mathrm{DR}}$ is a relative power measurement (and thus can be accurately calibrated), any systematic deviation of the scatter from the 1:1 line is attributed to the radar system gain. By adjusting the radar constant slightly (typically \pm 2 $\mathrm{dB}$ relative to its nominal value), the scatter can be made to lie along the 1:1 line. This method is used in this paper to derive the reflectivity $Z_{\mathrm{HH}}$ accurately from the CSUCHILL radar. In 1994, the radar was configured as a singletransmitter/single-receiver system with a high power polarization switch and thus was capable of $Z_{\mathrm{HH}}, Z_{\mathrm{DR}}, K_{\mathrm{DP}}$, and $\rho_{\mathrm{HV}}$ measurements without LDR. In 1995 and 1996, the two-transmitter/two-receiver configuration was used; the mean transmitted power from the two transmitters was continuously recorded. Thus, the radar constant was slightly different for the two configurations mainly because of the switch insertion loss and a finite receiver bandwidth loss due to use of narrower intermediate-frequency filters in the 1995 configuration. The radar constant adjustment procedure using $K_{\mathrm{DP}}$ thus used radar data from June 20, 1994, during a convective rain event and from June 2, 1995, also during a convective rain event. Visual observations during these two events confirmed that no frozen hydrometeors were contaminating the data. The radar constant established in 1995 was used for the 1996 data since there was essentially no change in the system configuration.

In this paper, we have used two Ka-band attenuationprediction schemes, one using $Z_{\mathrm{HH}}$ only and the other using $K_{\mathrm{DP}}$. We have not used the attenuation-prediction scheme of Goddard and Cherry [8] using both $Z_{\mathrm{HH}}$ and $Z_{\mathrm{DR}}$ because of contamination by frozen hydrometeors in the two convective cases analyzed. In light rain rates, it is known that the errors in attenuation prediction using $Z_{\mathrm{HH}}$ and $Z_{\mathrm{DR}}$ can at times become large, outweighing the added advantage of $Z_{\mathrm{DR}}$ over the simpler $k-Z_{\mathrm{HH}}$ method.

A radar-based attenuation model was developed from simulations that were obtained by varying the parameters of a gamma DSD. Using a Mie solution for spherical water particles, propagation variables such as Ka-band specific attenuation and S-band reflectivity were computed over a wide range of DSD parameters. In this case, a gamma DSD was used

$$
N(D)=N_{0} D^{m} e^{-\Lambda D}
$$

where

$$
\Lambda=\frac{3.67+m}{D_{0}}
$$

and $N(D)$, given in $\mathrm{mm}^{-1} \mathrm{~m}^{-3}$, is the number of drops per unit volume per unit size interval, $D$ is the equivalent drop size diameter in $\mathrm{mm}, N_{0}$ is given in $\mathrm{mm}^{-1-m} \mathrm{~m}^{-3}$, $D_{0}$ is the median drop size in $\mathrm{mm}$, and $m$ is the shape factor. The DSD triplets $\left(N_{0}, D_{0}, m\right)$ are varied as follows: $100 \leq N_{0} \leq 50000,1<D_{0} \leq 4$, and $0 \leq m \leq 5$. Results of the simulation are shown in Fig. 1. Each point on the

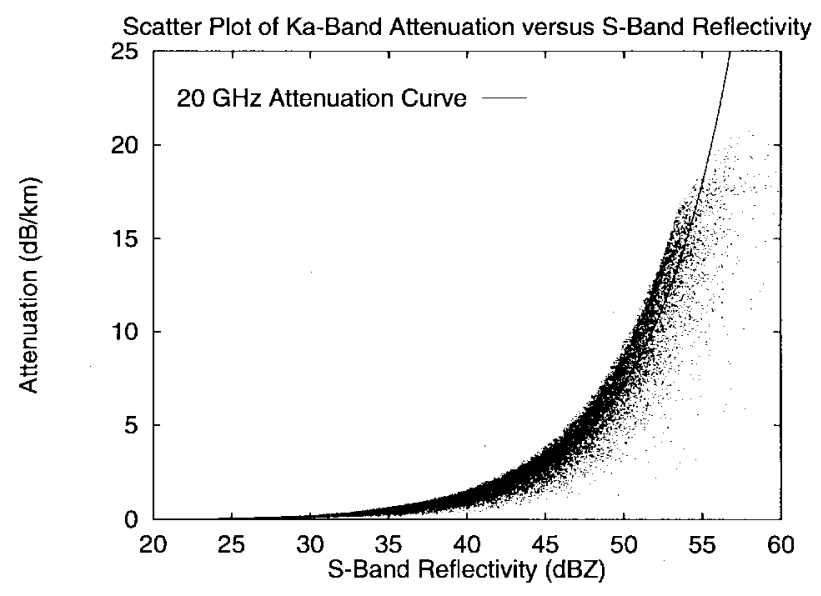

(a)

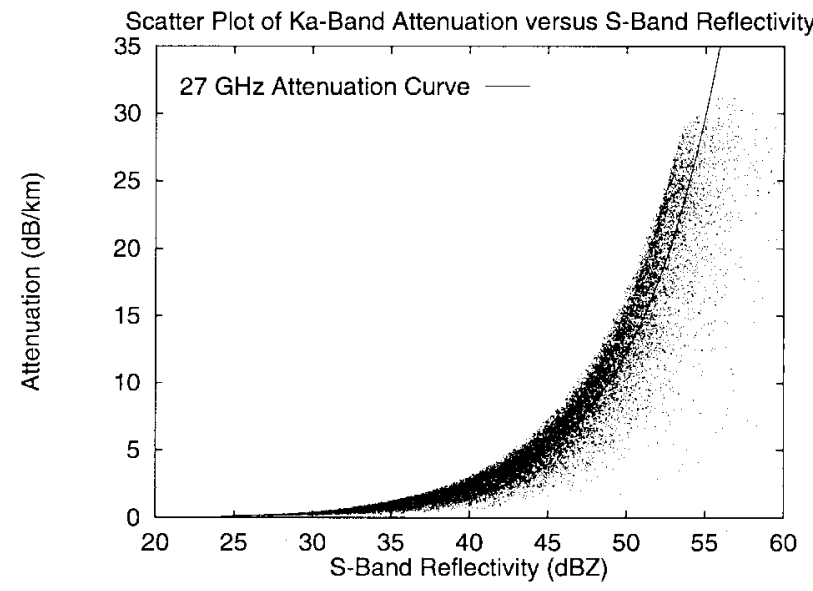

(b)

Fig. 1. Specific attenuation obtained using a Mie solution for spherical water particles. (a) $20 \mathrm{GHz}$ versus reflectivity at $3 \mathrm{GHz}$. (b) $27 \mathrm{GHz}$ versus reflectivity at $3 \mathrm{GHz}$. A gamma DSD is assumed. The solid line shows power law fit to the various gamma distributions.

Table 2 Values for a and b Given in (9)

\begin{tabular}{|c|c|c|}
\hline \hline Frequency & a & b \\
\hline $20 \mathrm{GHz}$ & $7.1 \times 10^{-4}$ & $8.0 \times 10^{-1}$ \\
\hline $27 \mathrm{GHz}$ & $1.7 \times 10^{-3}$ & $7.7 \times 10^{-1}$ \\
\hline \hline
\end{tabular}

scatter plot represents Ka-band specific attenuation and Sband reflectivity for a given DSD triplet with all points considered to be equiprobable. The entire range of triplets is representative of actual DSD's for a wide variety of rainfall events [18].

The S-band reflectivity/Ka-band specific attenuation curves are obtained by applying a power function fit to the simulated data. The equation that relates Ka-band specific attenuation to S-band reflectivity is given by

$$
k=a\left(Z_{\mathrm{HH}}\right)^{b}
$$

where $k$ is Ka-band specific attenuation and $Z_{\mathrm{HH}}$ is S-band reflectivity. Values for $a$ and $b$ are given in Table 2 .

To see the sensitivity of (9) to melting ice particles, simulations were also conducted using a two-layered Mie solution. Several scatter plots were obtained by simulating 


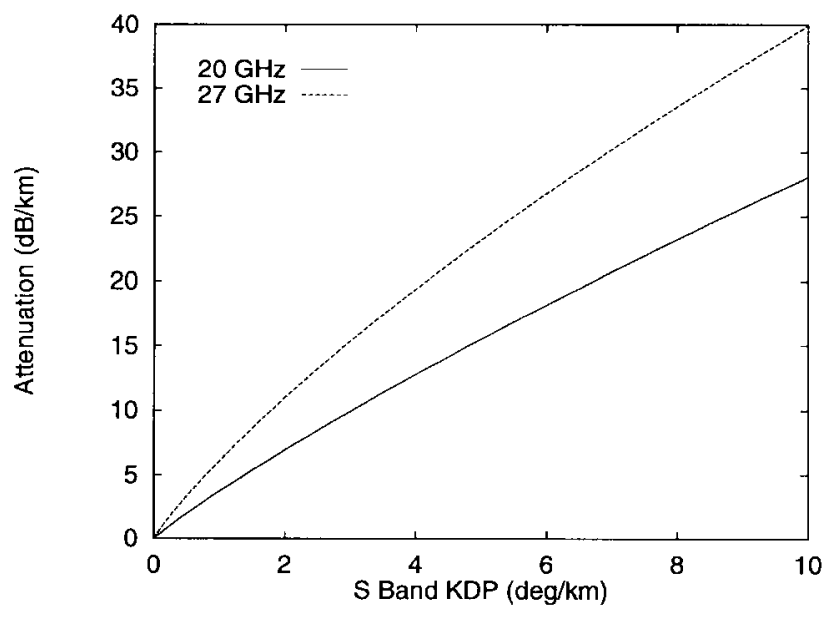

Fig. 2. Specific attenuation for 20 and $27 \mathrm{GHz}$ versus specific differential phase at $3 \mathrm{GHz}$ obtained via T-matrix and Mueller matrix solutions for oblate raindrops. An exponential DSD is assumed.

water-coated ice particles and varying the fraction of ice to water. The gamma DSD triplets were varied as before. Particles that were simulated ranged from spheres with small ice cores and a thick layer of water to spheres with large ice cores and a very thin layer of water. The derived attenuation curves in each case were almost identical to those shown in Fig. 1. Any variations noted fell well within the scatter of the DSD parameters for pure water. Thus, it appears that a separate treatment of melting ice hydrometeors may not be necessary in convective cases where melting graupel and hail are common. In stratiform cases, however, the bright-band attenuation is caused by melting snow and a separate model is generally used to arrive at the coefficients $a, b$. In this paper, we do not use separate bright-band model results for reasons mentioned earlier.

Attenuation curves relating Ka-band specific attenuation versus $\mathrm{S}$-band specific differential phase $K_{\mathrm{DP}}$ were also derived. The T-matrix solution [19]-[21] was used to obtain the scattering amplitudes for oblate raindrops ranging $1-8 \mathrm{~mm}$ in size. $\mathrm{S}$-band $K_{\mathrm{DP}}$ and specific attenuation at Ka-band were then computed from the Mueller matrix [22] averaged over an exponential DSD $(m=0$ in the gamma DSD). The attenuation curves shown in Fig. 2 were obtained by varying the DSD parameter $D_{0}$ while $N_{0}$ was fixed at $8000 \mathrm{~mm}^{-1} \mathrm{~m}^{-3}$. These curves are not very sensitive to variation in $N_{0}$ or $m$.

\section{DESCRIPTION OF PRECIPITATION EVENTS}

The Colorado Front Range experiences a variety of weather events throughout the year ranging from upslope rain conditions to winter storms producing wet snow and sleet to widespread convective episodes in the summer. Four summer events are presented in this section. These include two stratiform events with well-defined bright bands, where there was light-to-moderate precipitation uniformly covering a large area. Also presented are two convec-
ACTS Data - Convective Case

6/20/94 ACTS Propagation Data (CO)

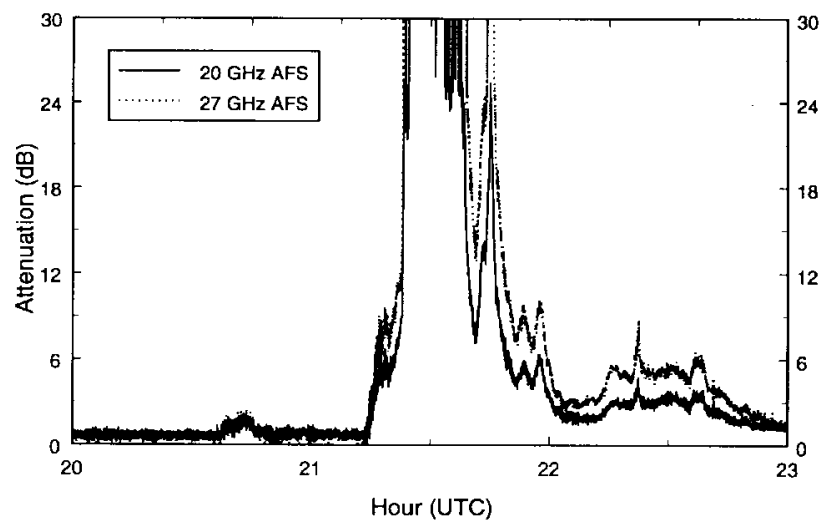

Fig. 3. CSU-APT attenuation measurements for the June 20, 1994, convective case. AFS refers to beacon attenuation with respect to free space.

tive cases, which are highly variable in nature and more localized to a particular region.

The events are presented in the order of occurrence and include a convective event on June 20, 1994, stratiform events on June 18, 1995, and July 6, 1996, and a convective event on July 7, 1996. For these four events, many radar scans were taken along the ACTS propagation path during the course of the precipitation event. The coordinates of the propagation path are $173^{\circ}$ from the north in the azimuthal direction and $43^{\circ}$ in elevation. For the first two events, the CSU-APT was collocated at the CSU-CHILL radar site in Greeley, CO. For the radar antenna diameter of $8.5 \mathrm{~m}$ and wavelength of $10 \mathrm{~cm}$, the $2 D^{2} / \lambda$ distance is $1.44 \mathrm{~km}$. We have observed using the copolar correlation coefficient $\left(\rho_{\mathrm{HV}}\right)$ measurement that data from the first $2 \mathrm{~km}$ in range is contaminated and unusable in retrieval of attenuation. Also, for elevation angles $\geq 20^{\circ}$, the polarimetric variables such as $Z_{\mathrm{DR}}$ and $\Phi_{\mathrm{DP}}$ decrease rapidly with increasing angle in a uniform rain medium. Therefore, elevation angle correction must be performed when using the attenuation-prediction equations based on $K_{\mathrm{DP}}$, which are valid only at low elevation angles $\left(\leq 20^{\circ}\right)$. To avoid these complications, the APT was moved $13 \mathrm{~km}$ south of the radar. The move also allows for greater use of the radar polarimetric parameters in identifying precipitation types. At the new site, a radar elevation angle of $20^{\circ}$ intersects the APT slant path at a height of approximately $7 \mathrm{~km}$, well above the melting layer.

The first case was a strong attenuation event that occurred on June 20, 1994, causing a signal loss at $27 \mathrm{GHz}$ for approximately $15 \mathrm{~min}$. The $20-\mathrm{GHz}$ signal bounced in and out of lock several times but only for very short durations. Fig. 3 shows the measured APT data for both the 20- and 27-GHz channels. Attenuation levels actually reached the $30-\mathrm{dB}$ level at both frequencies before the signal began to cut in and out. The event started at approximately 21:14 coordinated universal time (UTC), with large attenuation present at both frequencies until 22:01 UTC. Concurrent radar data were also taken for the June 20 rain event. Fig. 4 shows a range-height indicator (RHI) plot taken at 21:32 

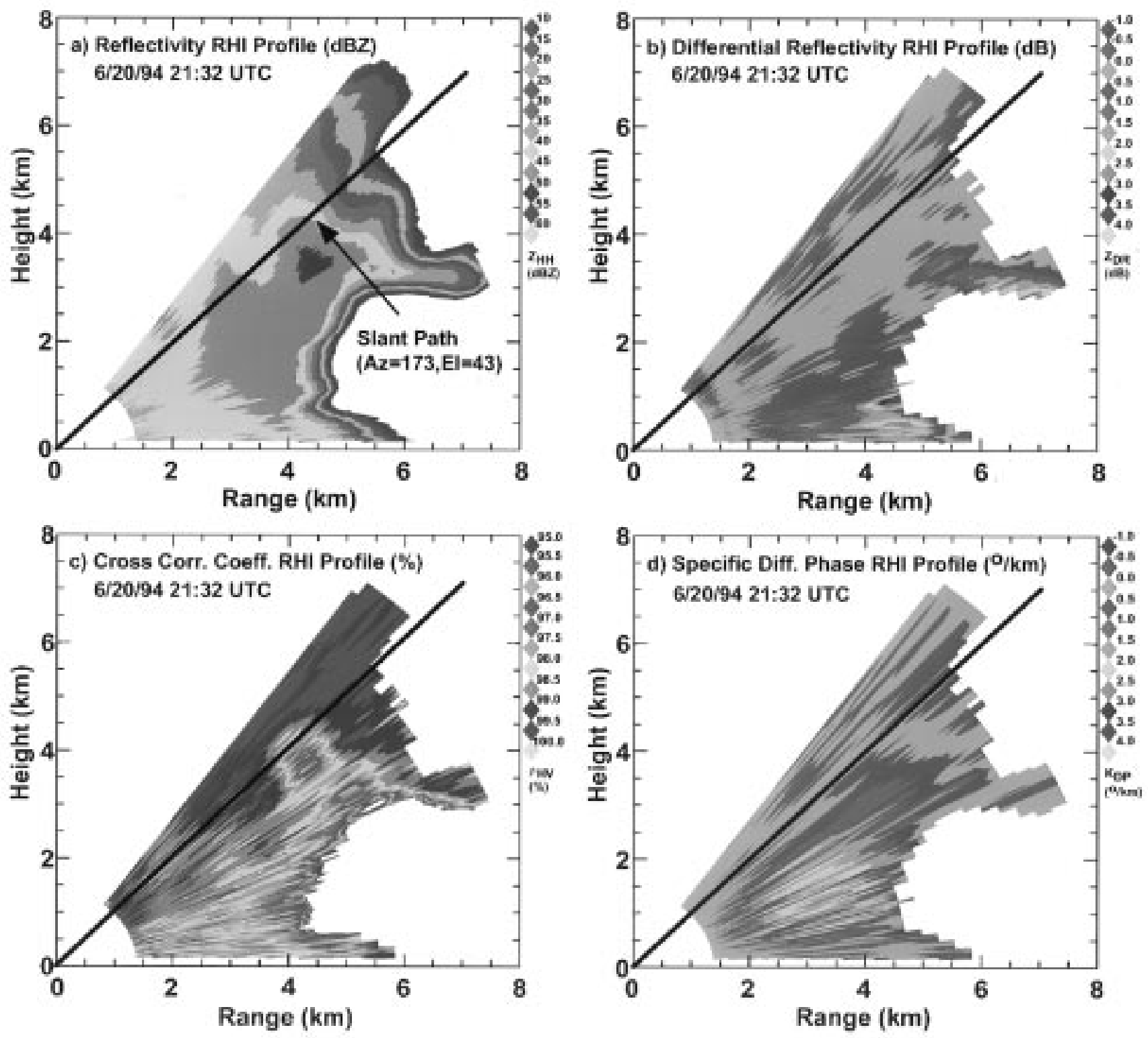

Fig. 4. RHI scan for the June 20, 1994 convective case. (a) Horizontal reflectivity $Z_{\mathrm{HH}}$ (dBZ). (b) Differential reflectivity $Z_{\mathrm{DR}}(\mathrm{dB})$. (c) The cross correlation coefficient $\rho \mathrm{HV}$ (given as a percentage). (d) Specific differential phase $K_{\mathrm{DP}}(\mathrm{deg} / \mathrm{km})$. The scan is taken along the direction of the ACTS propagation path. The CSU-APT elevation angle is $43^{\circ}$. The radar and CSU-APT are located at the origin with the height given in $\mathrm{km}$ along the $y$-axis. Distance away from the radar along the $172^{\circ}$ azimuthal angle is given in $\mathrm{km}$ along the $x$-axis.

UTC. Reflectivity values [Fig. 4(a)] along the propagation path were on the order of $45 \mathrm{dBZ}$; at $6.5 \mathrm{~km}, Z_{\mathrm{H}}$ reached a maximum of $54 \mathrm{dBZ}$. Values of $Z_{\mathrm{DR}}$ [Fig. 4(b)] were on the order of $0.3-0.5 \mathrm{~dB}$ along the propagation path. In the area of peak reflectivity, the $Z_{\mathrm{DR}}$ values were approximately $0.5-0.8 \mathrm{~dB}$. These low values of $Z_{\mathrm{DR}}$ combined with the high reflectivity values give an indication that there were possibly tumbling hailstones in that particular region. Fig. 4(c) shows a dip in the copolar correlation coefficient in the region of low $Z_{\mathrm{DR}}$ and high $Z_{\mathrm{H}}$ while along the rest of the propagation path it was 0.98 or greater, which is typical for a pure rain or ice medium. There were also measurable values of specific differential phase $K_{\mathrm{DP}}$ at 6.5 $\mathrm{km}$, as shown in Fig. 4(d). Values of $K_{\mathrm{DP}}$ along the slant path were approximately $0.5-0.6{ }^{\circ} \mathrm{km}^{-1}$. The presence of $K_{\mathrm{DP}}$ and a minimum in the correlation coefficient at $6.5 \mathrm{~km}$ is an indication that this region was an area of mixed-phase hydrometeors. The region likely consisted of tumbling hailstones along with water-coated ice particles [13]. This region of mixed phase occurred right along the ACTS propagation path.

The next event to be examined was a stratiform event that occurred on June 18, 1995. As shown in Fig. 5, this was actually a stratiform case with some embedded convection in the initial stages of the event. Peak values of attenuation measured by the APT were around $10 \mathrm{~dB}$ for the 27 $\mathrm{GHz}$ signal and $5 \mathrm{~dB}$ for the $20-\mathrm{GHz}$ signal during the embedded convective part of the event. In the more stable 


\section{ACTS Data - Stratiform Case}

6/18/95 ACTS Propagation Data (CO)
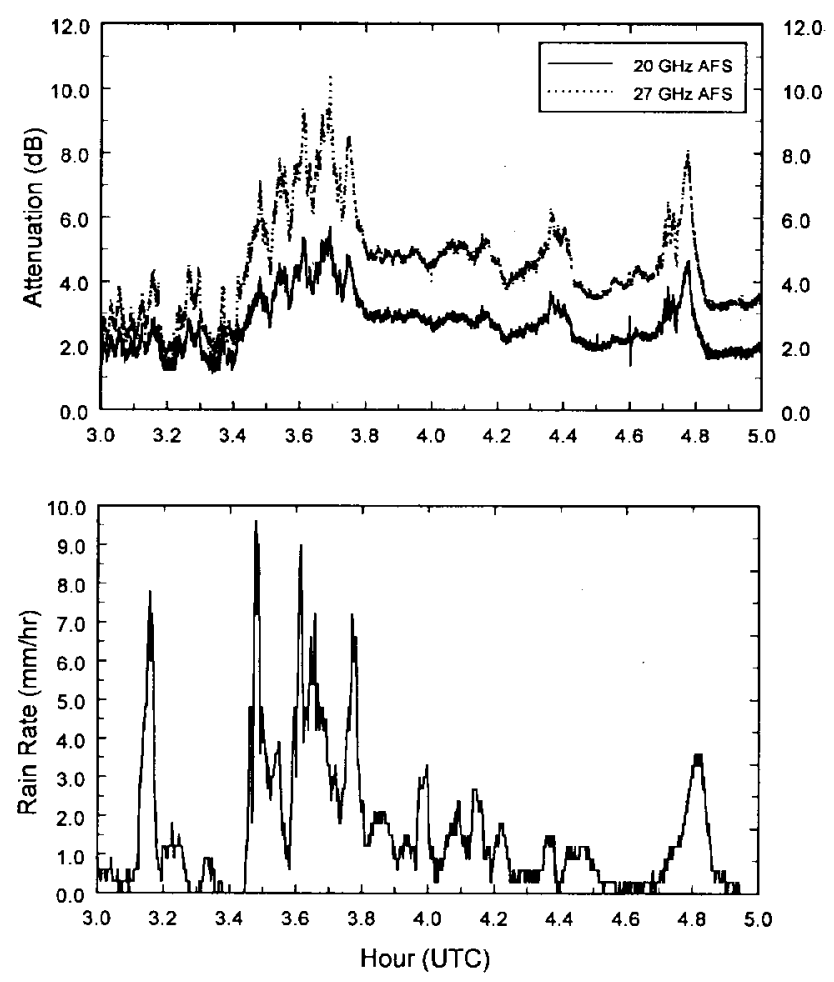

Fig. 5. CSU-APT beacon attenuation measurements and the corresponding rain rate (from a gauge at the site) for the June 18, 1995 stratiform case. AFS refers to beacon attenuation with respect to free space.

stratiform region, the attenuation was approximately 5 and $3 \mathrm{~dB}$ for the $27-$ and $20-\mathrm{GHz}$ signals, respectively. The rain rates (from a capacitive rain gauge located at the radar site) for this event are also given, with peak rates of only 6-9 $\mathrm{mmh}^{-1}$ during the portion of the event with embedded convection and $1-3 \mathrm{mmh}^{-1}$ during the stratiform-only time of the event.

An RHI plot taken at 4:21 UTC is shown in Fig. 6. Fig. 6(a) shows a well-defined reflectivity bright band. The horizontal reflectivity had values of $40-45 \mathrm{dBZ}$ in this region, which was at a height of approximately $2.45 \mathrm{~km}$. Below the bright band, values of $30 \mathrm{dBZ}$ were seen along the propagation path. The enhanced reflectivity was due to the aggregation of falling ice particles and an increase in the dielectric constant as the particles began to melt [23]. Values of $Z_{\mathrm{DR}}$ reached a peak of 1.0-1.5 dB toward the bottom of the melting layer, with values of $0.5-0.8$ $\mathrm{dB}$ in the rain region below the melting layer, as seen in Fig. 6(b). This enhanced region of $Z_{\mathrm{DR}}$ can be attributed to several factors - the aggregates were beginning to melt, they were becoming more oblate, and they had stabilized into a preferential orientation. The increasing dielectric constant also contributed significantly to the increase of differential reflectivity.

The copolar correlation coefficient $\rho_{\mathrm{HV}}$ shown in Fig. 6(c) also exhibited a well-defined layer near the melting level. The correlation coefficient is dependent on both the size and shape distributions of the hydrometeors. It is also affected by differential phase shift upon backscatter due to Mie scattering; in addition, mixtures of hydrometeor types contribute to a reduction in $\rho_{\mathrm{HV}}$ values. This is due to the wider range in the size and shape distributions for two types of particles as compared to one [23]. For a bright-band case, the minimum for $\rho_{\mathrm{HV}}$ typically occurs between the peak values of $Z_{H}$ and $Z_{\mathrm{DR}}$ [23]. Typical values for $\rho_{\mathrm{HV}}$ in a medium that consists of only one type of hydrometeor, such as rain, are 0.98 or greater. In this case, values of $\rho_{\mathrm{HV}}$ in the bright band were 0.93-0.94, which indicates that a wide distribution of particle types and sizes were in this region. The final radar parameter given in this RHI plot [Fig. 6(d)] is the linear depolarization ratio, which had values up to $-18 \mathrm{~dB}$ in the melting layer due to the wet snow particles [13]. Below the bright band, the LDR values are in the range of -30 to $-32 \mathrm{~dB}$, which are typical for light rain, while above the bright band the values are around -26 to $-28 \mathrm{~dB}$, which are typical for ice particles.

The third precipitation event was a stratiform case that occurred on July 6, 1996. The CSU-APT (then located 13 $\mathrm{km}$ south of the radar) measured attenuation is shown in Fig. 7. Attenuation levels at $27 \mathrm{GHz}$ peaked at approximately $11-12 \mathrm{~dB}$, while at $20 \mathrm{GHz}$ the peak levels of attenuation were $7-8 \mathrm{~dB}$. The radar representation of this event is illustrated by the RHI plot taken at 3:31 UTC, as shown in Fig. 8. The measured reflectivity in the region of the bright band was about 35-40 dBZ, while values of 30-35 dBZ were seen along the propagation path below the reflectivity bright band. The height of the reflectivity bright band was approximately $2.8 \mathrm{~km}$. $Z_{\mathrm{DR}}$ values near the bottom of the melting layer were approximately $0.8-1.2$ $\mathrm{dB}$, with values of $0.7-0.8 \mathrm{~dB}$ below the melting layer. The $\rho_{\text {HV }}$ data exhibited the typical decrease in magnitude in the melting region, where values dropped to 0.93 . The LDR had values up to $-18 \mathrm{~dB}$ in the melting layer with similar values above and below the bright band, as in the previous stratiform event.

The final event was a convective case that occurred on July 7, 1996. As in the previous convective case, both beacon signals were interrupted due to attenuation. The 27$\mathrm{GHz}$ beacon signal was out for $20 \mathrm{~min}$ and the $20-\mathrm{GHz}$ beacon signal was interrupted for approximately $11 \mathrm{~min}$. The CSU-APT measured attenuation is shown in Fig. 9. Note the loss of beacon signals at both frequencies during the central part of the event. The symmetric nature of the attenuation event is suggestive of a strong storm cell advecting across the slant path. The RHI plot for this event, shown in Fig. 10, was taken at 2:04 UTC. There were fairly high reflectivities present along a significant portion of the slant path. Values ranged from 50-55 dBZ along the first $1.5 \mathrm{~km}$ of the slant path to $35-40 \mathrm{dBZ}$ along the slant path as far out as $13.5 \mathrm{~km}$. Values for $Z_{\mathrm{DR}}$ are on the order of 3.0-3.5 $\mathrm{dB}$ up to a height of $1.5 \mathrm{~km}$, where a distinct transition occurred. These high $Z_{\mathrm{DR}}$ values indicate large oblate raindrops. Above the $1.5-\mathrm{km}$ altitude, the rapid decrease in $Z_{\mathrm{DR}}$ to $0 \mathrm{~dB}$ was caused by nearly spherical or randomly oriented ice hydrometeors. Therefore, the high 

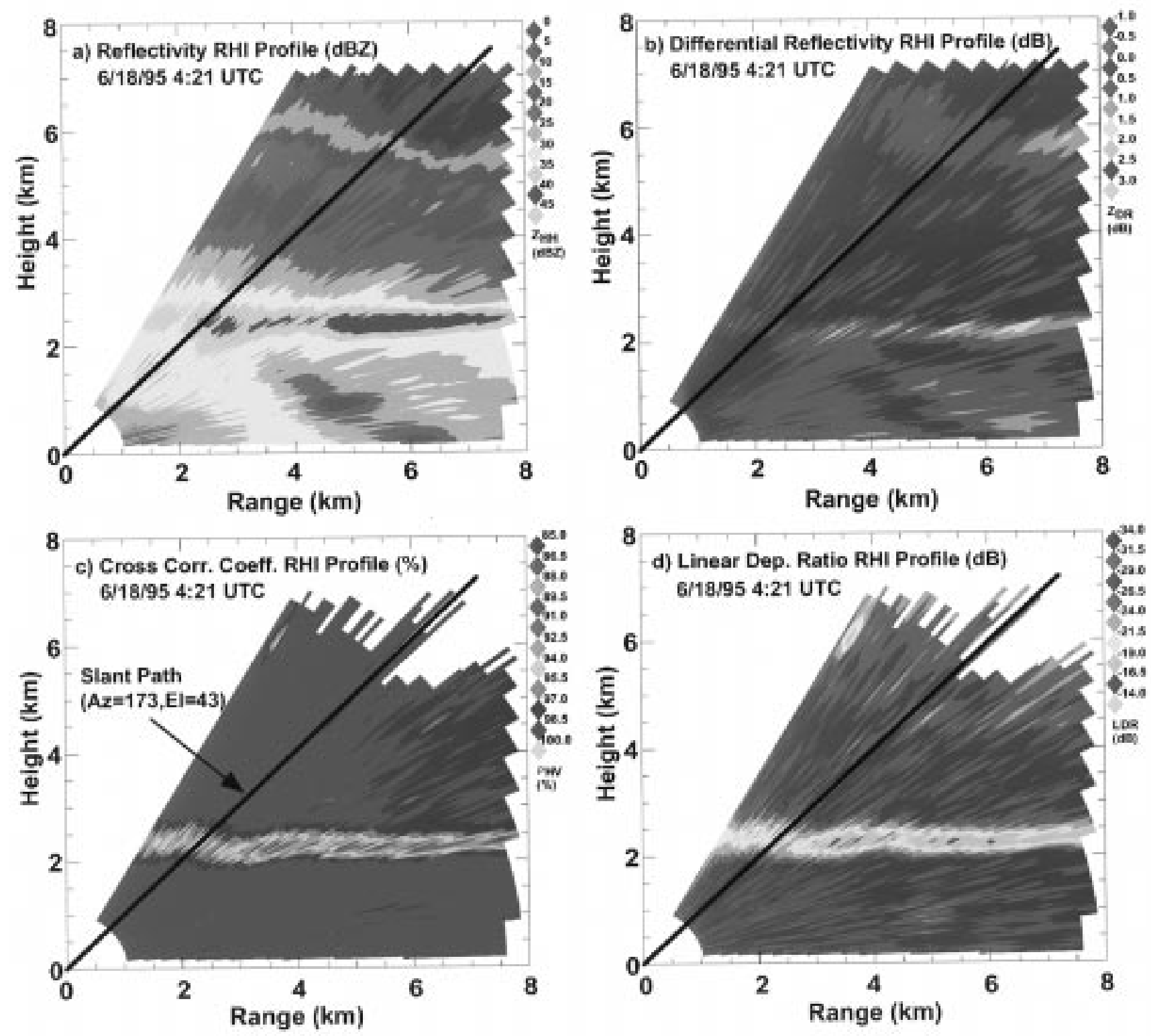

Fig. 6. RHI scan for the June 18,1995 stratiform case. (a) Horizontal reflectivity $Z_{\mathrm{HH}}$ (dBZ). (b) Differential reflectivity $Z_{\mathrm{DR}}(\mathrm{dB})$. (c) The cross-correlation coefficient $\rho \mathrm{HV}$ (given as a percentage). (d) The linear depolarization ratio $(\mathrm{dB})$. The scan is taken along the direction of the ACTS propagation path. The CSU-APT elevation angle is $43^{\circ}$. The radar and CSU-APT are located at the origin with the height given in $\mathrm{km}$ along the $y$-axis. Distance away from the radar along the $172^{\circ}$ azimuthal angle is given in $\mathrm{km}$ along the $x$-axis.

reflectivities that were seen aloft, which occurred along the slant path, were due to ice particles and did not contribute significantly to the attenuation. The bottom panels show $\rho_{\mathrm{HV}}$ [Fig. $\left.10(\mathrm{c})\right]$ and $K_{\mathrm{DP}}$ [Fig. 10(d)]. A distinct change in $\rho_{\mathrm{HV}}$ can be noted at around $1.5 \mathrm{~km}$ in altitude from 0.99 above to $0.97-0.98$ below. These lowered $\rho_{\mathrm{HV}}$ values are indicative of raindrops mixed with small hailstones. The $K_{\text {DP }}$ panel shows a region of high values in the range $1.0-3.5^{\circ} \mathrm{km}^{-1}$ below $1.5-\mathrm{km}$ altitude. The high rain rates $\left(40-120 \mathrm{mmh}^{-1}\right)$ and high $Z_{\mathrm{DR}}$ values suggest that the precipitation was dominated by raindrops.

The next step in the analysis is to use the radar information as input to the attenuation model on a case-by-case basis. Results using the S-band reflectivity/Ka-band specific attenuation model are presented in Section IV for the four case studies.

\section{EXPERIMENTAL RESULTS}

\section{A. Predicted Ka-Band Attenuation Estimates}

Attenuation estimates have been computed using the specific attenuation models derived in Section II. For the two stratiform events described in the previous section, only reflectivity data were used to predict Ka-band attenuation. Specific differential phase was not used due to the low rain rates and the measurement accuracy of $K_{\mathrm{DP}}( \pm 0.3$ 
ACTS Data - Stratiform Case

7/6/96 ACTS Propagation Data (CO)

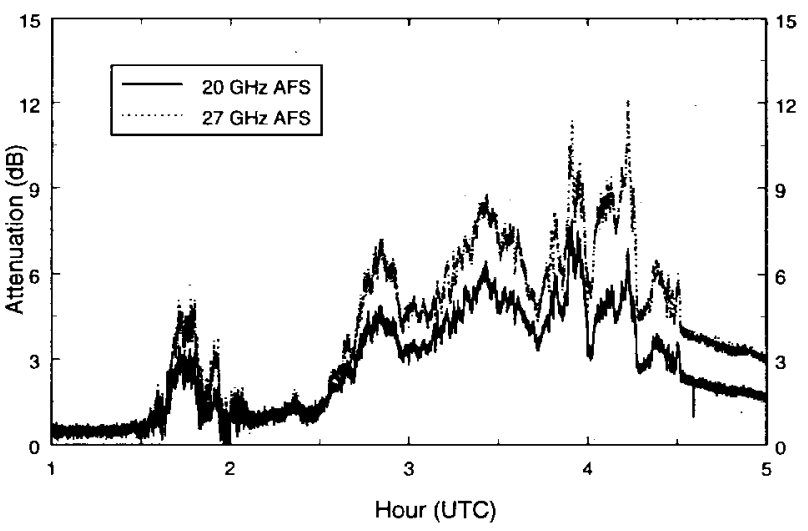

Fig. 7. CSU-APT beacon attenuation measurements for the July 6, 1996 stratiform case. AFS refers to beacon attenuation with respect to free space.

${ }^{\circ} \mathrm{km}^{-1}$ ). For the two convective cases both reflectivity and $K_{\mathrm{DP}}$ were used to predict specific attenuation at $\mathrm{Ka}-$ band. The polarimetric parameters $Z_{\mathrm{DR}}, K_{\mathrm{DP}}$, and $\rho_{\mathrm{HV}}$ were used to determine subjectively the effective length of the attenuation path for all events. For the first two case studies, these polarimetric parameters are dependent on the elevation angle of the radar. Therefore, a correction factor was used to obtain values of $K_{\mathrm{DP}}$ for an elevation angle of $0^{\circ}$. The corrected values are used as input to the attenuation model. For the final two case studies, no elevation angle correction was needed.

For the cases when the CSU-APT was collocated at the radar site (June 20, 1994 and June 18, 1995), radar scans were taken directly along the ACTS propagation path with a range resolution of $150 \mathrm{~m}$. Reflectivity data were taken at the $150-\mathrm{m}$ increments and used to determine the corresponding 20- and $27-\mathrm{GHz}$ attenuation estimates from (9). The Ka-band attenuation estimates were then multiplied by the appropriate distance along the propagation path. For the two cases where the CSU-APT was located $13 \mathrm{~km}$ south of the radar site (July 6 and 7, 1996), radar scans were taken at several azimuth angles close to the $173^{\circ}$ azimuth of the ACTS propagation path. The radar also scanned in elevation from 0 to $30^{\circ}$. For each elevation scan at a particular azimuth angle, only the radar data that intersected the CSU-APT slant path were used in obtaining attenuation estimates.

For the June 20, 1994, convective case, 43 radar scans were taken throughout the duration of the event. Ka-band attenuation estimates were derived from S-band reflectivity data using the procedure described above. For this particular event, S-band $K_{\text {DP }}$ data were also used to derive Ka-band attenuation estimates. The results are shown in Fig. 11.

As seen in Fig. 11, the CSU-CHILL reflectivity-based attenuation estimates follow the attenuation measurements obtained from the APT very closely. The maximum difference is about $5 \mathrm{~dB}$, while for the most part the CSU-CHILLderived estimates are within $1-2 \mathrm{~dB}$ of those measured by the CSU-APT. The attenuation estimates derived from $K_{\text {DP }}$ data alone grossly underestimated the attenuation caused by this event. This may be explained by examining Fig. 12, a scatter plot of the APT measured $20-\mathrm{GHz}$ attenuation versus the one-way differential phase $\left(\Phi_{\mathrm{DP}} / 2\right)$ measured by the CSU-CHILL radar.

One-way differential phase and its range derivative $K_{\mathrm{DP}}$ are sensitive to the oblateness of raindrops. Therefore, the difference seen between the maximum and minimum values of the fitted curve in Fig. 12 is caused by the presence of various numbers and sizes of oblate raindrops present in the propagation path. If the hydrometeors in the slant path were composed of only raindrops, the $y$-intercept of the fitted curve would be close to zero; however, if the fitted curve is extended back to $\Phi_{\mathrm{DP}}=0$, the $y$-intercept is at $23.97 \mathrm{~dB}$. This indicates that a large amount of attenuation was due to effectively spherical, water-coated ice particles for which $K_{\mathrm{DP}}$ is zero. By effectively spherical we also include tumbling or quasi-random orientation of particles for which $f_{\mathrm{HH}} \approx f_{\mathrm{VV}}$ in (3). That $K_{\mathrm{DP}}$ data alone grossly underestimates the attenuation is an indication that watercoated, effectively spherical ice particles (hailstones) could have been present throughout a large part of the propagation path during this event.

Considering this to be the case, the next step is to determine the reflectivity due only to the water-coated, effectively spherical ice particles along the propagation path from the total reflectivity measured by the CSU-CHILL radar. This is done using the difference reflectivity $Z_{\mathrm{DP}}$ to determine the ice fraction content in the radar resolution volume at each $150-\mathrm{m}$ increment along the propagation path [13]. We define $Z_{\mathrm{DP}}=10 \log \left(Z_{\mathrm{HH}}-Z_{\mathrm{VV}}\right)$ so that effectively spherical particles would have $Z_{\mathrm{HH}} \approx Z_{\mathrm{VV}}$ giving no contribution to $Z_{\mathrm{DP}}$, similar to $f_{\mathrm{HH}} \approx f_{\mathrm{VV}}$ in (3) giving no contribution to $K_{\mathrm{DP}}$. Oblate raindrops, however, will have $Z_{\mathrm{HH}}>Z_{\mathrm{VV}}$ depending on their $Z_{\mathrm{DR}}$ values. In addition, for raindrops only there is a nearly linear relation between $Z_{\mathrm{DP}}$ and $Z_{\mathrm{HH}}^{\mathrm{rain}}$ called the rain line, which is experimentally determined. The presence of effectively spherical particles with raindrops causes the $Z_{\mathrm{HH}}$ to deviate from the rain line at a given $Z_{\mathrm{DP}}$. This deviation can be related to the reflectivity-weighted ice fraction $f=Z_{\mathrm{HH}}^{\mathrm{ice}} /\left(Z_{\mathrm{HH}}^{\mathrm{ice}}+Z_{\mathrm{HH}}^{\mathrm{rain}}\right)$ [13]. Once the ice fraction content is determined, the reflectivity due only to the water-coated ice particles, $Z_{\mathrm{HH}}$, can be determined. The $\mathrm{S}$-band reflectivity/Ka-band attenuation model is then used with $Z_{\mathrm{HH}}^{\mathrm{ice}}$ as the input to determine the attenuation at 20 and $27 \mathrm{GHz}$ for the effectively spherical, water-coated hail particles. The attenuation due to only the oblate raindrops is computed using the $\mathrm{S}$-band $K_{\mathrm{DP}} / \mathrm{Ka}$-band attenuation model.

The combined results are shown in Fig. 11; for the most part, the CSU-CHILL-derived attenuation estimates using $K_{\mathrm{DP}}$ and $Z_{\mathrm{HH}}^{\text {ice }}$ are within $1-2 \mathrm{~dB}$ of the attenuation values obtained by the CSU-APT. The $27-\mathrm{GHz}$ results are shown in Fig. 13. The 27-GHz signal was interrupted for approximately $16 \mathrm{~min}$ during this event. At approximately 21:39 UTC, the 27-GHz signal was reacquired and, as seen 

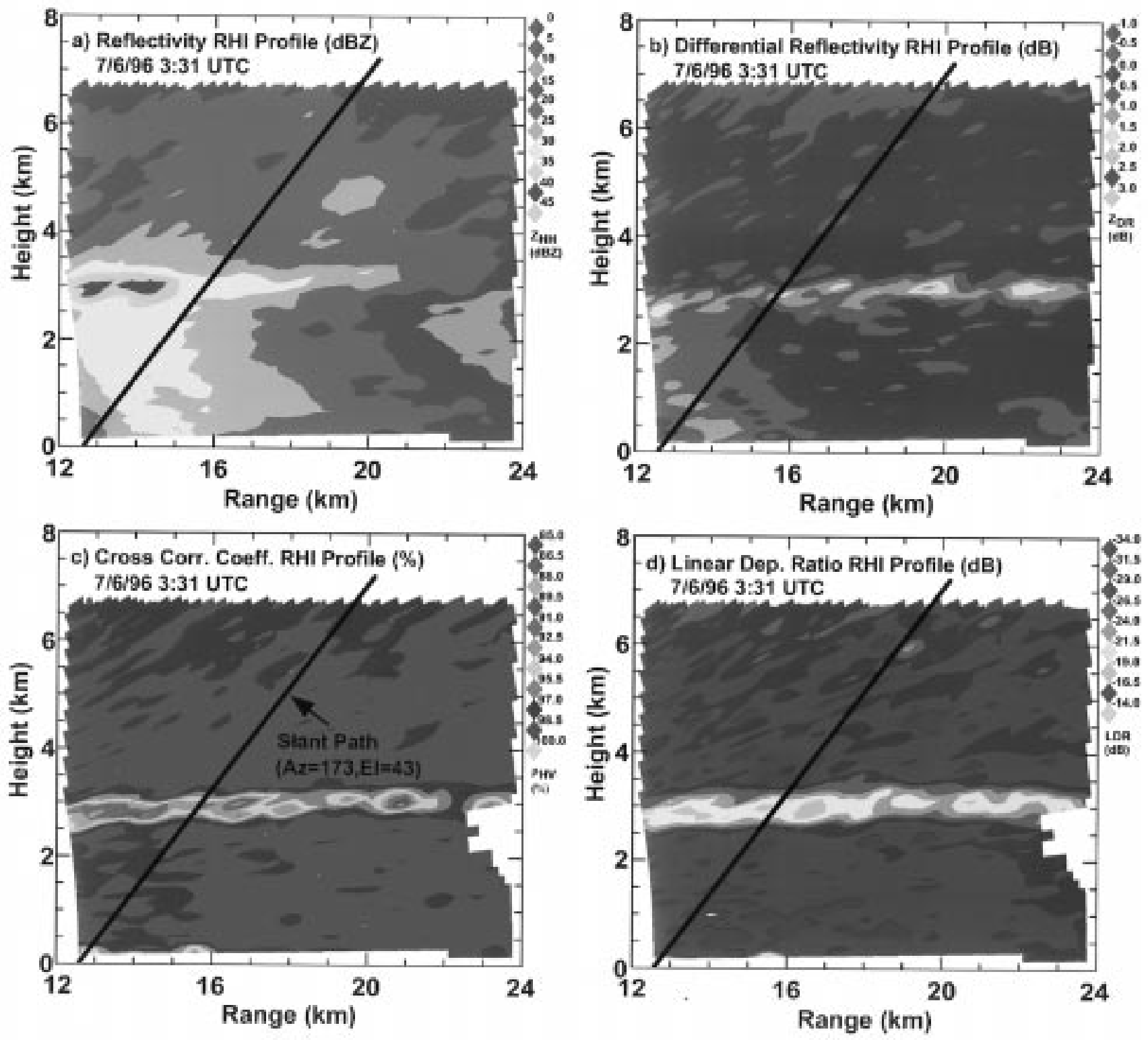

Fig. 8. RHI scan for the July 6, 1996 stratiform case. (a) Horizontal reflectivity $Z_{\mathrm{HH}}$ (dBZ). (b) Differential reflectivity $Z_{\mathrm{DR}}(\mathrm{dB})$. (c) The cross-correlation coefficient $\rho_{\mathrm{HV}}$ (given as a percentage). (d) The linear depolarization ratio $(\mathrm{dB})$. The CSU-APT elevation angle is $43^{\circ}$. The APT is located $13 \mathrm{~km}$ south from the radar site. Height in $\mathrm{km}$ is along the $y$-axis. Distance away from the radar is given in $\mathrm{km}$ along the $x$-axis.

in Fig. 13, the CHILL attenuation estimates are very close to the APT measured attenuation.

The results obtained by using the different polarimetric parameters available from the CHILL radar in this case are very encouraging. This is a good example of how the different parameters can be used to determine the nature of precipitation particles along the propagation path and predict Ka-band attenuation using S-band radar data.

Results for the June 18, 1995 stratiform case are shown in Fig. 14. The attenuation estimates obtained from the S-band reflectivity data follow the trend of the beacon attenuation estimates. The radar attenuation estimates, however, are off by an average value at both frequencies of approximately $1.8 \mathrm{~dB}$ at $20 \mathrm{GHz}$ and $3.0 \mathrm{~dB}$ at $27 \mathrm{GHz}$. This is also seen in the July 6, 1996 stratiform case, as shown in Fig. 15. Here, the reflectivity-based attenuation estimates underestimate the APT attenuation estimates by $2 \mathrm{~dB}$ at $20 \mathrm{GHz}$ and 3 $\mathrm{dB}$ at $27 \mathrm{GHz}$. Because of the frequency dependence of the underestimates, it is unlikely that they can be attributed to a systematic error in the S-band radar constant, i.e., the absolute calibration of the radar. A simple increase in Sband reflectivity by a few $\mathrm{dB}$ would be inconsistent not only with the frequency dependence but also with the difference in underestimates between the convective and stratiform portions of the same event (see Fig. 14). An explanation for the underestimation will be presented in Section IV-B.

The final case studied is the July 7, 1996 convective event. Results for this event are shown in Fig. 16. For 


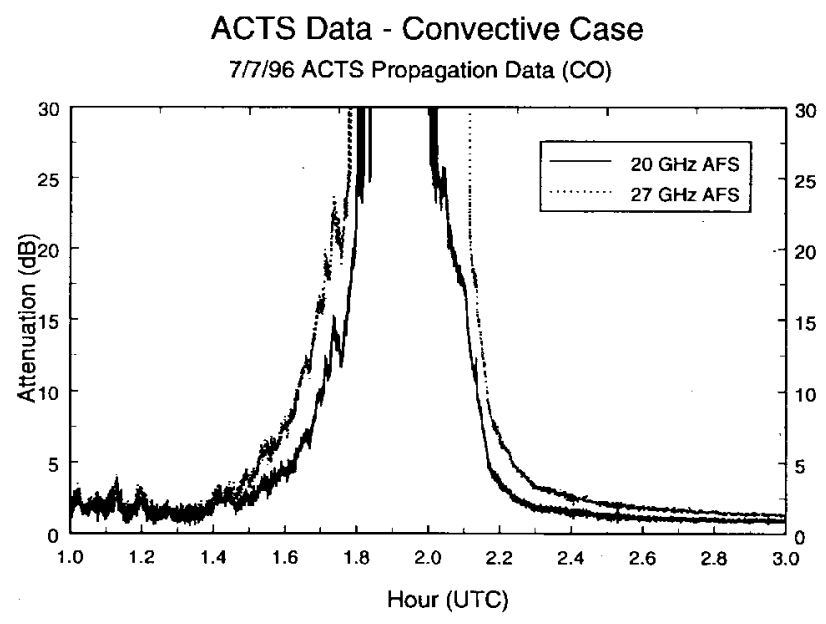

Fig. 9. CSU-APT beacon attenuation measurements for the July 7, 1996 convective case. AFS refers to beacon attenuation with respect to free space.

a large portion of the time that the radar was collecting data, both beacon signals were not being received due to attenuation. As in the previous convective case, there is good agreement between the CSU-APT measured attenuation estimates and the Ka-band attenuation estimates derived from $\mathrm{S}$-band reflectivity data. For the most part, the differences are on the order of 3-5 dB and the trend is for the reflectivity-based attenuation estimates to overestimate that of the measured attenuation. Results using $K_{\mathrm{DP}}$ data to derive Ka-band attenuation estimates via Fig. 2 are also shown in Fig. 16. Here the predicted attenuation was closer to the CSU-APT measured attenuation with a general trend slightly to underestimate the measured values by $1-2 \mathrm{~dB}$. A scatter plot of measured Ka-band attenuation versus Sband one-way differential phase for this event is shown in Fig. 17. There is very good correlation between Ka-band attenuation and S-band differential phase. For this event, the $\mathrm{y}$-intercept of the fitted line is $4.3 \mathrm{~dB}$ for $20 \mathrm{GHz}$ and $6.9 \mathrm{~dB}$ for $27 \mathrm{GHz}$, much smaller than in Fig. 12. Again, for a pure rain event, the y-intercept for the fitted curves should be close to zero. It is also likely that precipitation in this event was composed predominantly of raindrops mixed with peato-marble-sized hail. This was observed at the surface by CSU personnel in mobile chase vans who intercepted the storm cell about 10-20 km west of the APT site.

Last, a scatter plot of measured Ka-band attenuation versus mean rain rate is shown in Fig. 18. The mean rain rate is computed using $K_{\mathrm{DP}}$ values that are available along the ACTS slant path and using the following relationship

$$
\bar{R}=\left\langle 40.5 K_{\mathrm{DP}}^{0.845}\right\rangle
$$

The brackets \langle\rangle indicate that an average has been taken over all the points available along the slant path. Therefore, $\bar{R}$ is the mean rain rate that occurs along the slant path at a specific instant of time. As expected, there is very good correlation between measured Ka-band attenuation and the mean rain rate.

\section{B. Source of Underestimation in Predicted Ka-band Attenuation Estimates for the Stratiform Events}

Underestimation of Ka-band attenuation levels from Sband radar data has been noted in previous studies [24] and the differences have been attributed to gaseous attenuation that is not measured at the S-band frequencies. In Colorado, however, only $0.4-0.5 \mathrm{~dB}$ of attenuation can be attributed to the gaseous constituents. These values were determined by using radiometrically derived attenuation as well as observations from radiosonde data taken from the Denver area. Gaseous attenuation and attenuation from cloud liquid water will contribute partially to the differences seen in the two cases described, but this contribution is small. Another reason may be due to the bright-band attenuation's not being accounted for properly in our model. Our model, however, would actually overpredict the bright-band attenuation and thus would not explain the radar-derived underestimates. Our hypothesis for the radar underestimation of attenuation is that water collecting on the antenna surface in the form of droplets may cause appreciable amounts of attenuation due to absorption and/or reflection and defocusing effects. This possibility is examined by conducting a water-spraying experiment. Under clear sky conditions, a garden hose was used to simulate the effect of rainfall on the antenna surface only, the feed horn surface only, and both the antenna surface and feed horn surfaces simultaneously. The results of spraying the antenna surface alone are shown in Fig. 19.

The antenna surface was continuously sprayed with water starting at 21:36:00 UTC (21.60 h) and ending at 21:37:46 UTC $(21.63 \mathrm{~h})$. This is noted by the sustained peak attenuation in Fig. 19. After the spraying had stopped there was a quick decrease in attenuation until 21:38:09 UTC (21.64 h). After this point, the attenuation continued to decrease but at a much slower rate. This measured attenuation is due to water standing on the antenna surface in the form of small droplets or beads. This beading effect will also occur during rain events and is dependent on the rain rate.

To check if the same type of frequency dependence is exhibited in the water-spraying test as that seen in the differences between the attenuation estimates at 20 and $27 \mathrm{GHz}$ shown in Figs. 14 and 15, the instantaneous attenuation ratios were compared. The attenuation ratio for the water-spraying test and for the June 18, 1995 stratiform case are shown in Fig. 19. Once the spraying had stopped, the attenuation ratio between the June 18 event and the attenuation ratio computed from attenuation due to water droplets on the antenna surface for the most part are identical. This gives a good indication that most of the difference seen between the attenuation estimates derived from S-band reflectivity data and CSU-APT attenuation estimates for the June 18, 1995 and July 6, 1996 events were due to water droplets that formed on the antenna surface.

The amount of attenuation caused by droplets forming on the antenna surface is dependent on the rain rate, the wind direction and speed, and the type of event, and therefore is 

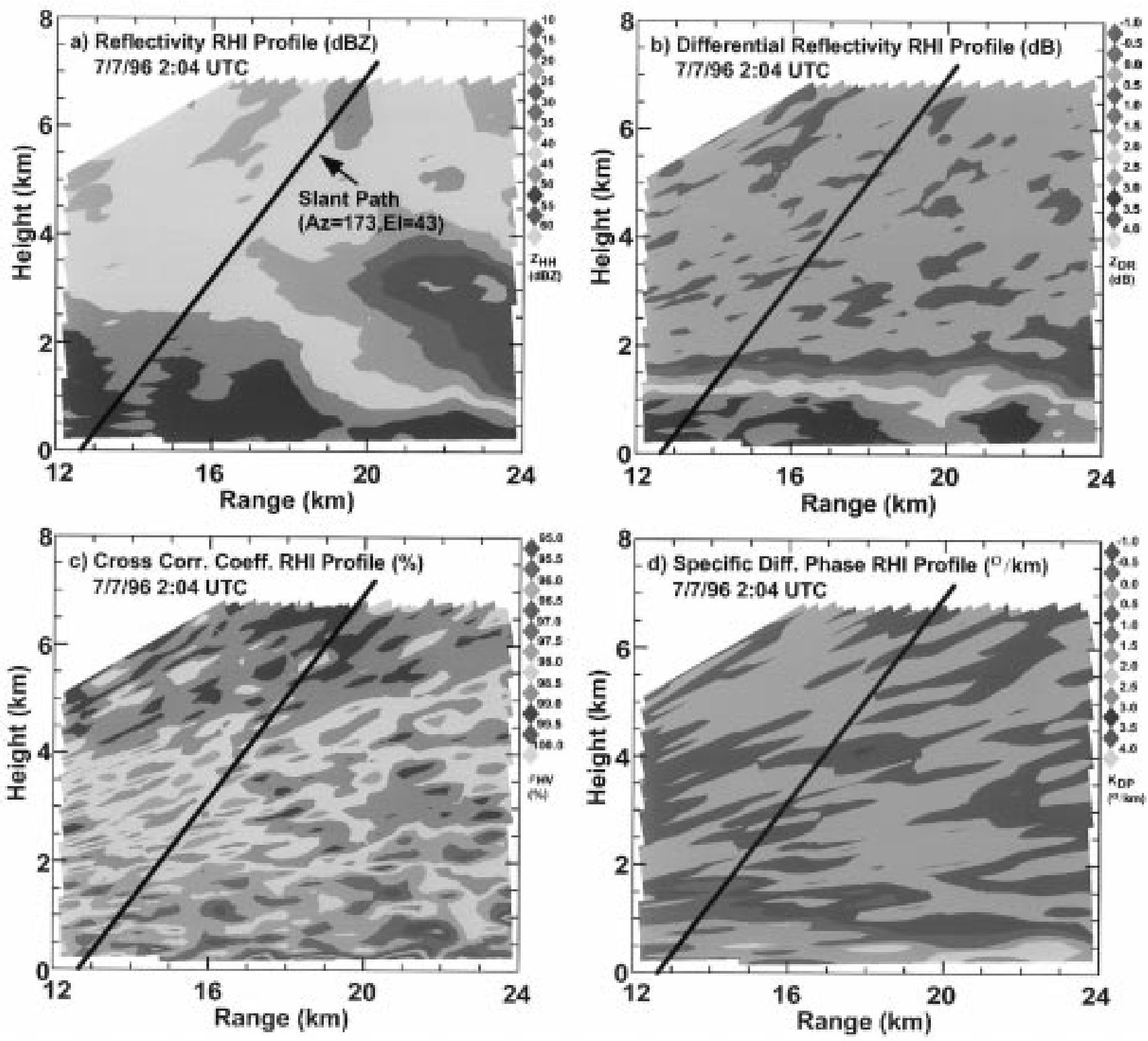

Fig. 10. RHI scan for the July 7, 1996 convective case. (a) Horizontal reflectivity $Z_{\mathrm{HH}}$ (dBZ). (b) Differential reflectivity $Z_{\mathrm{DR}}(\mathrm{dB})$. (c) The cross-correlation coefficient $\rho_{\mathrm{HV}}$ (given as a percentage). (d) Specific differential phase $K_{\mathrm{DP}}(\mathrm{deg} / \mathrm{km})$. The CSU-APT elevation angle is $43^{\circ}$. The APT is located $13 \mathrm{~km}$ south from the radar site. Height in $\mathrm{km}$ is along the $y$-axis. Distance away from the radar is given in $\mathrm{km}$ along the $x$-axis.

not a systematic error that can be easily removed. While a hydrophobic solution was applied to the antenna surface to minimize this effect, water droplets still collected on the antenna surface. Several additional experiments were conducted by spraying the antenna surface with water. It was found that $1-9 \mathrm{~dB}$ of attenuation could occur at $27 \mathrm{GHz}$ and $1-6 \mathrm{~dB}$ of attenuation at $20 \mathrm{GHz}$ just due to a water buildup on the antenna surface only. Previous experiments that involved the wetting of the antenna surface reported minimal attenuation due to water on the antenna surface, particularly in stratiform rain events where rain rates were low [25], [26]. Theoretical loss calculations due to uniform water layers on radomes and antenna reflecting surfaces have been performed by Blevis [27]; however, he did not consider the practically important case of droplets on the antenna surface. Subsequent controlled experiments carried out at CSU by wetting the APT antenna surface showed that for rain rates of $20-30 \mathrm{mmh}^{-1}$, the attenuation level for $20 \mathrm{GHz}$ was about $4 \mathrm{~dB}$ and for $27 \mathrm{GHz}$ approximately $6 \mathrm{~dB}$ [28]. Results from spraying the feed horn surface show that the beading effect does not occur with the same magnitude as seen on the antenna surface. In addition, the feed horn surface dries much more quickly than the antenna surface. Several ACTS propagation sites, including Oklahoma, Florida, and CSU, have experienced attenuation of beacon power levels on the order of $2-3 \mathrm{~dB}$ just from dew condensing on the antenna surface. The structure of the antenna surface is the most probable cause for the 


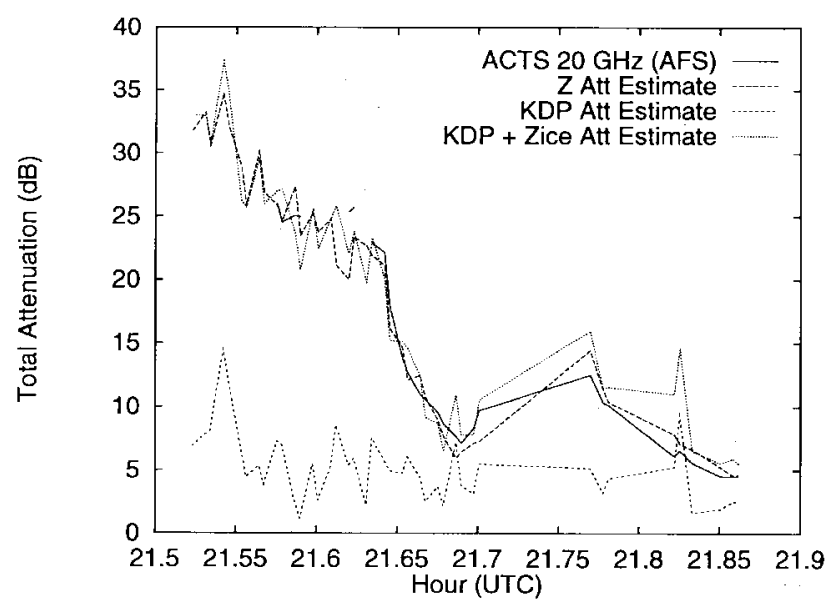

Fig. 11. Comparison of measured CSU-APT attenuation (solid line) and 20-GHz attenuation estimates derived from CSU-CHILL data for June 20, 1994 event.

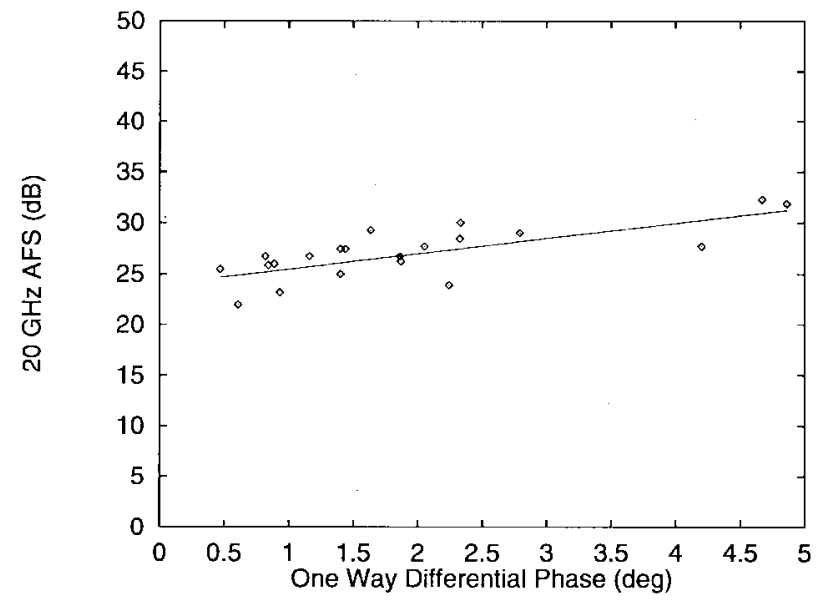

Fig. 12. 20-GHz attenuation versus one-way differential phase for the June 20, 1994 event. The solid line shows (least-squares) straight-line fit.

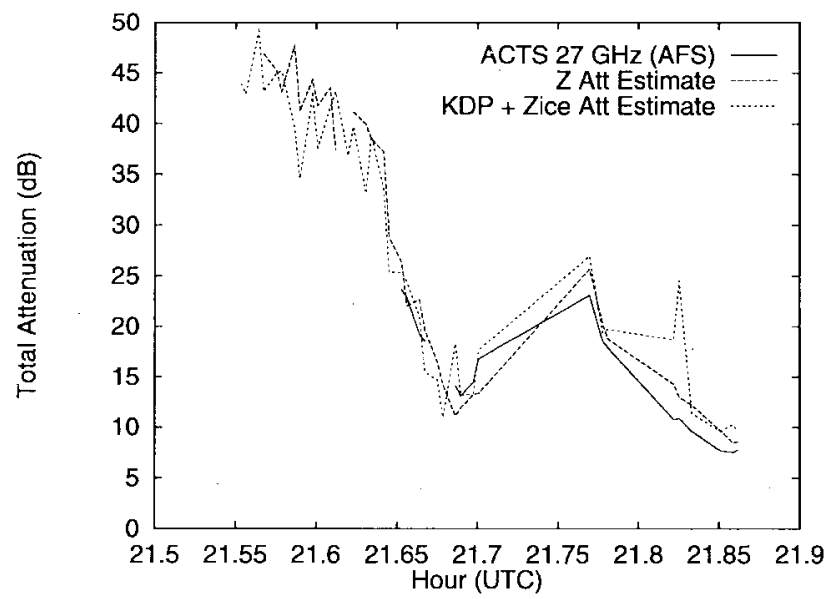

Fig. 13. Comparison of measured CSU-APT attenuation (solid line) and 27-GHz attenuation estimates derived from CSU-CHILL data for the June 20, 1994 event.

magnitude of the effective loss that is attributed to antenna wetting in this experiment. This particular antenna surface is textured with features that are $0.08-\mathrm{mm}$ deep, which makes it very conducive to retaining water on its surface
June 18, 1995 Stratiform Case

Comparison of CSU-CHILL and CSU-APT Attenuation Estimates
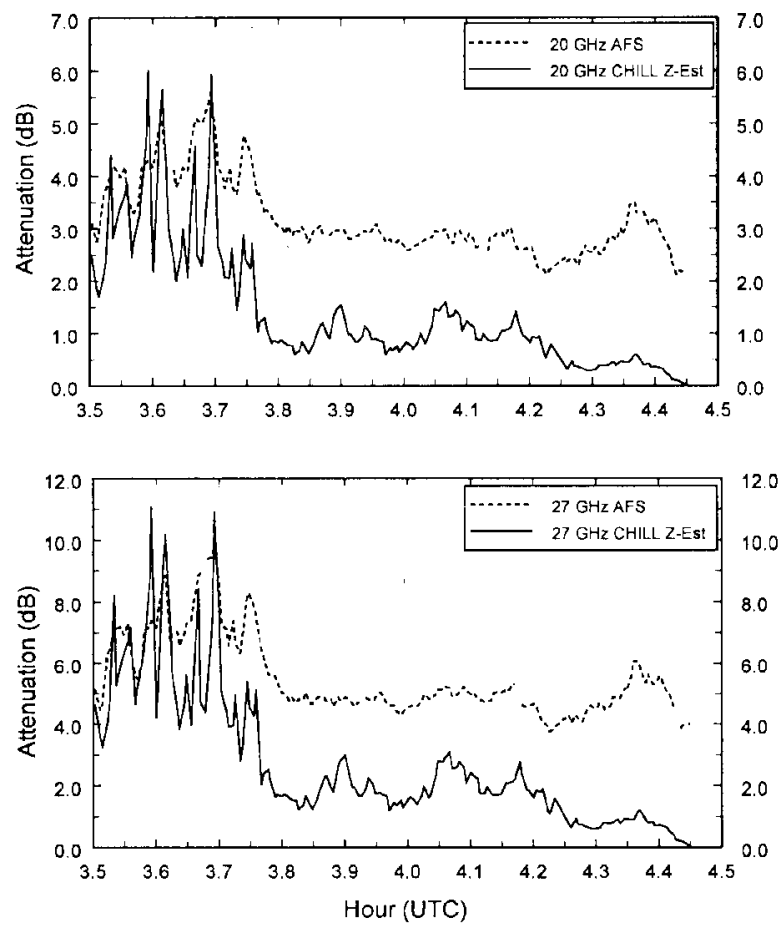

Fig. 14. Comparison of measured CSU-APT attenuation at 20 and $27 \mathrm{GHz}$ (dashed lines) with attenuation estimates derived from CSU-CHILL S-band reflectivity data (solid lines). For the June 18, 1995 stratiform case.

July 6, 1996 Stratiform Case

Comparison of CSU-CHILL and CSU-APT Attenuation Estimates
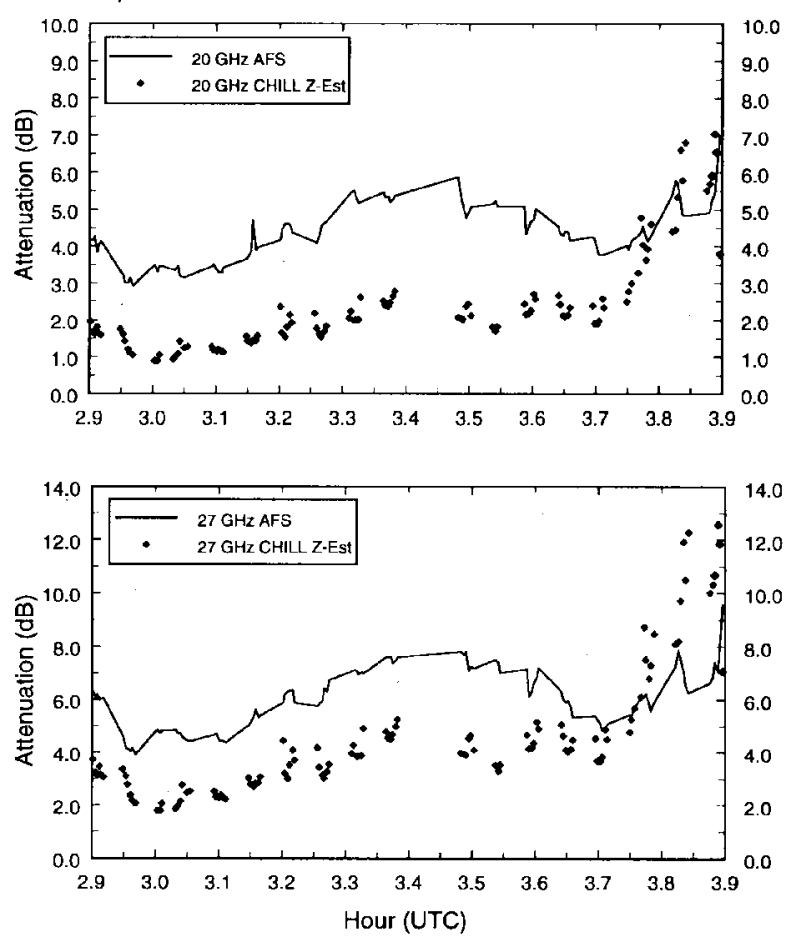

Fig. 15. Comparison of measured CSU-APT attenuation at 20 and $27 \mathrm{GHz}$ (solid lines) with attenuation estimates derived from CSU-CHILL S-band reflectivity data. For the July 6, 1996 stratiform case. 
July 7, 1996 Convective Case

Comparison of CSU-CHILL and CSU-APT Attenuation Estimates
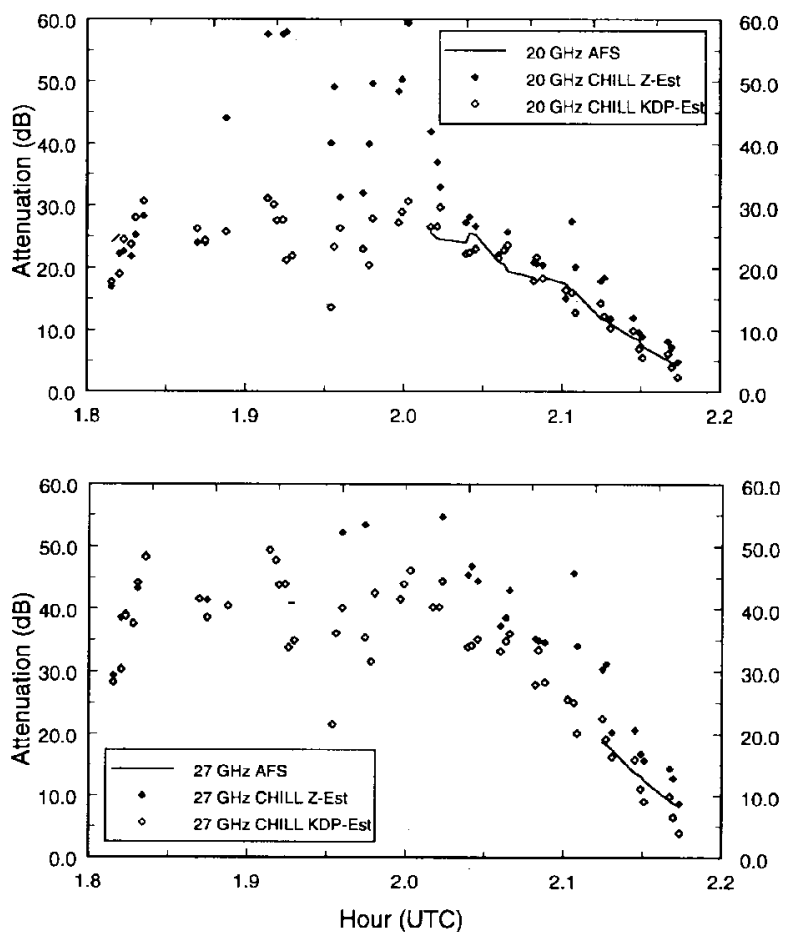

Fig. 16. Comparison of measured CSU-APT attenuation at 20 and $27 \mathrm{GHz}$ (solid lines) with attenuation estimates derived from CSU-CHILL S-band reflectivity and $\mathrm{K}_{\mathrm{DP}}$ data. For the July 7, 1996 convective case.
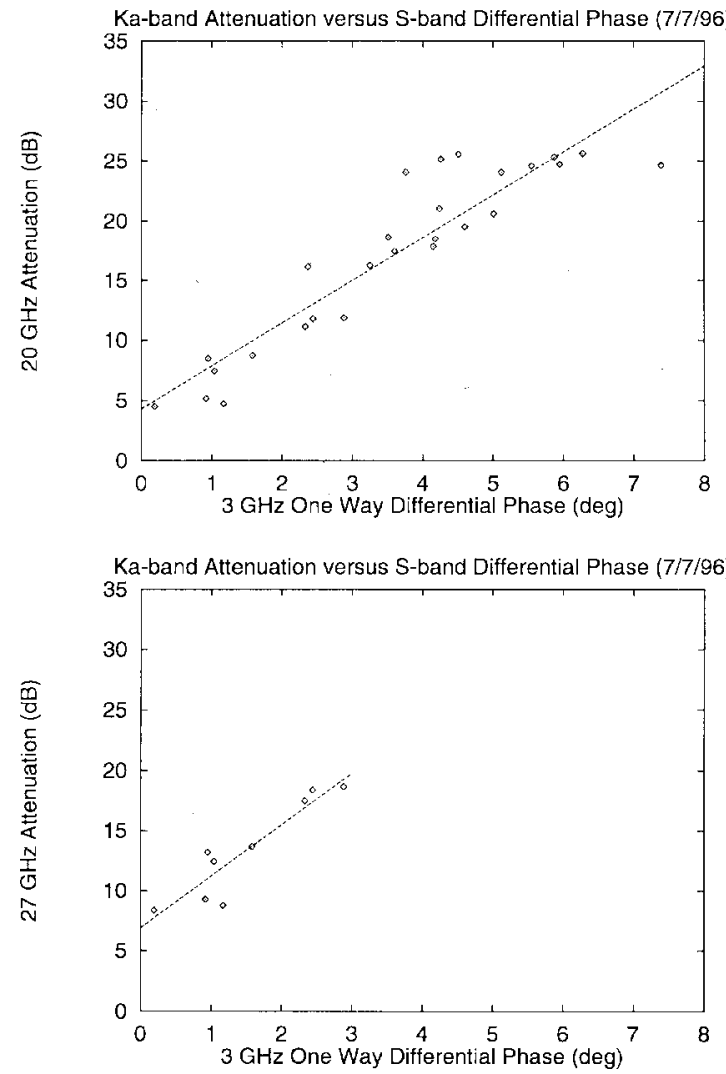

Fig. 17. Attenuation for 20 and $27 \mathrm{GHz}$ versus one-way differential phase at $3 \mathrm{GHz}$ for the July 7, 1996, convective case. Dashed line shows (least-squares) straight-line fit.
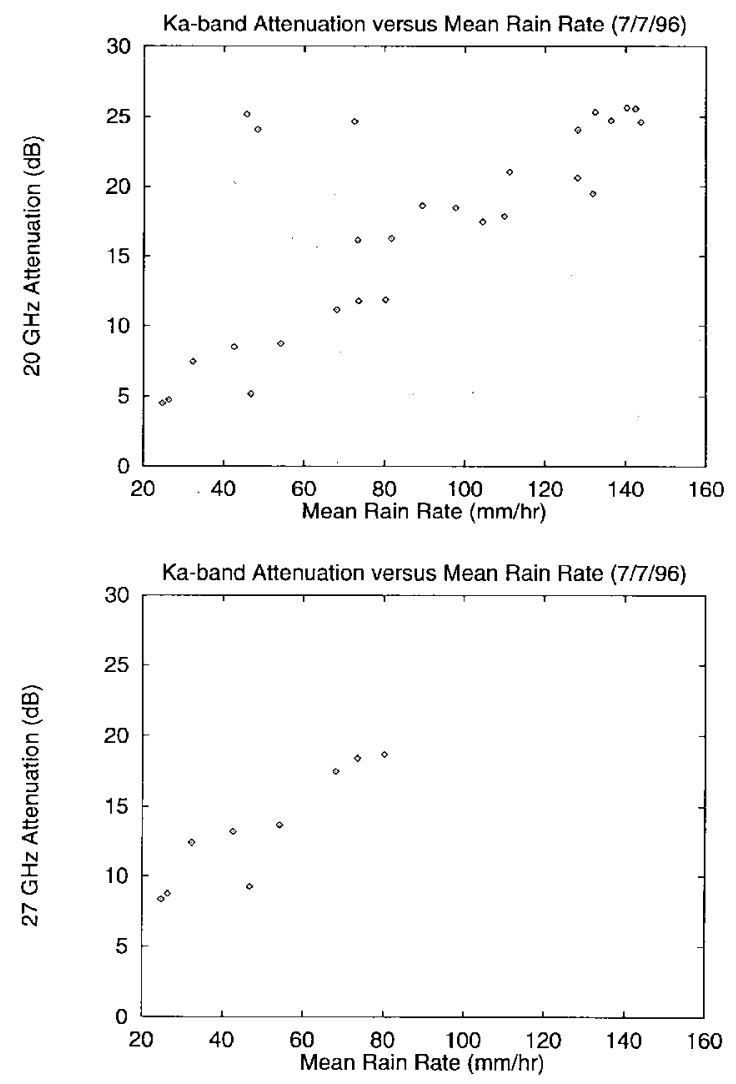

Fig. 18. Attenuation for 20 and $27 \mathrm{GHz}$ versus mean rain rate for the July 7, 1996, convective case. Mean rain rate is derived from average $K_{\mathrm{DP}}$ values along the ACTS beacon path.

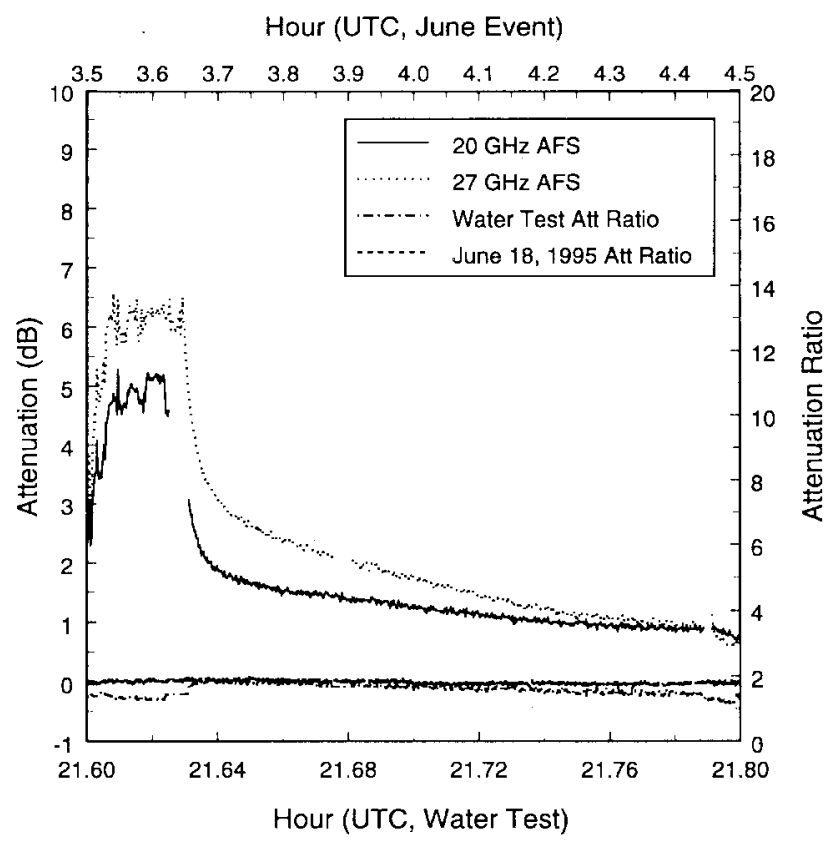

Fig. 19. Water-spray test results. Water was sprayed only on the antenna surface. Beacon attenuations for 20 and $27 \mathrm{GHz}$ are shown, along with the instantaneous attenuation ratio for the two frequencies $(20 \mathrm{GHz}$ is the base frequency).

in the form of droplets. While it may be difficult to remove these effects, they do need to be taken into account for model development and in statistical analysis. 


\section{CONCLUSIONS}

Due to the high demand for satellite communications, already overused portions of the frequency spectrum (Cand Ku-bands) are becoming even more crowded. This necessitates looking at less crowded areas of the spectrum, such as the Ka-band frequencies. To study satellite communications at these frequencies, NASA launched ACTS. The ACTS is an experimental satellite being used to conduct communication and propagation experiments using new Kaband technology. At Ka-band, weather events can have an adverse affect on the signal being propagated through the atmosphere. Therefore, propagation effects at these frequencies must be studied. This paper has outlined one of the research areas that has been undertaken at CSU during the first two years to further the understanding of Ka-band propagation effects.

Attenuation models were developed to relate S-band radar reflectivity and differential propagation phase to Kaband specific attenuation. Several case studies were presented to illustrate the attenuation models. These included a very strong convective storm that occurred on June 20, 1994, two stratiform events that occurred on June 18, 1995 and July 6, 1996, and a convective event that occurred on July 7, 1996. Polarimetric radar data collected by the CSU-CHILL radar for each of these events were used to provide additional data on the precipitation events. Good agreement between the CSU-APT measured Ka-band attenuation estimates and the predicted Ka-band attenuation was achieved for the two convective events. The two stratiform events produced interesting results that have been noted in previous low-attenuation events, i.e., underestimation of Ka-band attenuation estimates derived from S-band reflectivity data. The most probable cause for this underestimation was discussed in Section IV. Water droplets standing on the antenna surface during very intense precipitation can cause a maximum of $6 \mathrm{~dB}$ of attenuation at $20 \mathrm{GHz}$ and $9 \mathrm{~dB}$ of attenuation at $27 \mathrm{GHz}$. The results obtained here and by the other ACTS propagation experimenters will greatly enhance the knowledge of using Ka-band frequencies for satellite communications.

\section{ACKNOWLEDGMENT}

The authors greatly appreciate the assistance rendered by P. Kennedy, K. Pattison, and B. Bowie of the CSUCHILL staff in data collection and by Dr. E. Mueller and D. Brunkow, who spearheaded the radar modifications to a two-transmitter/two-receiver system.

\section{REFERENCES}

[1] D. C. Cox, "An overview of the Bell Laboratories 19 and 28 GHz COMSTAR beacon propagation experiments," Bell Syst. Tech. J., vol. 57, no. 4, pp. 1231-1240, 1978.

[2] J. Goldhirsh, "A review on the application of nonattenuating frequency radars for estimating rain attenuation and space diversity performance polarimetric radar signatures of precipitation at S- and C-bands," IEEE Trans. Geosci. Electron., vol. GE-17, Oct. 1979.

[3] J. P. V. Poiares Baptista, Reference Book on Attenuation Measurement and Prediction, vol. 1. Noordwijk, The Netherlands: OPEX, ESA-WPP 083, 1994.
[4] W. L. Stutzman, T. Pratt, C. Nunnally, R. Nealy, W. Remaklus, A. Predoehl, and D. Gaff, "ACTS propagation terminal hardware description report," Virginia Polytechnic Institute and State University, Tech. Rep. EE SATCOM 93-9, June 1993.

[5] R. K. Crane, "A two-component rain model for the prediction of attenuation statistics," Radio Sci., vol. 17, no. 6, pp. 1371-1387, 1982.

[6] Y. M. M. Antar, A. Hendry, J. Schlesak, and R. Olsen, "Measurements of ice depolarization at $28.56 \mathrm{GHz}$ using the COMSTAR beacon simultaneously with a $16.5 \mathrm{GHz}$ polarization diversity radar," IEEE Trans. Antennas Propagat., vol. AP-30, Sept 1982.

[7] S. M. Cherry, J. W. F. Goddard, and M. P. M. Hall, "Use of dual-polarization radar data for evaluation of attenuation on a satellite-to-earth path," Ann. Telecommun., vol. 36, pp. 33-39, 1981.

[8] J. W. F. Goddard and S. M. Cherry, "The ability of dual polarization radar (copolar linear) to predict rainfall rate and microwave attenuation," Radio Sci., vol. 19, no. 1, pp. 201-208, 1984.

[9] R. E. Marshall, T. Pratt, E. A. Manus, D. P. Stapor, and J. H. Andrews, "S-band radar differential reflectivity measurements in multiple polarization planes along satellite slant paths," Radio Sci., vol. 19, no. 1, pp. 109-114, 1984.

[10] J. W. F. Goddard, J. D. Eastment, and M. Thurai, "The Chilbolton advanced meteorological radar: A tool for multidisciplinary atmospheric research," Electron. and Commun. Eng. J., vol. 6, no. 2, pp. 77-86, Apr. 1994.

[11] J. P. V. Poiares Baptista, OPEX: Reference Book on Radar, vol. 4. Noordwijk, The Netherlands: OPEX, 1994.

[12] E. A. Mueller and D. Staggs, "Capabilities of the CHILL radar after update," Preprints, 23rd Conf. Radar Meteorol., American Meteorological Society, Snowmass, CO, 1986.

[13] R. J. Doviak and D. S. Zrnic, Doppler Radar and Weather Observations. San Diego, CA: Academic, 1993.

[14] V. N. Bringi, L. Liu, P. C. Kennedy, V. Chandrasekar, and S. A. Rutledge, "Dual multiparameter radar observations of intense convective storms: The 24 June 1992 case study," Meteorol. Atmos. Phys., vol. 59, pp. 3-31, 1996.

[15] K. V. Beard and C. Chuang, "A new model for the equilibrium shape of raindrops," J. Atmos. Sci., vol. 44, no. 11, pp. 1509-1524, 1987.

[16] A. R. Jameson, "Theoretical analysis and meteorological interpretation of the role of the raindrop shape on microwave attenuation and propagation phase shift: Implication for the radar measurement of rain," J. Atmos. Oceanic Technol., vol. 6, pp. 76-88, 1989.

[17] V. Chandrasekar, V. N. Bringi, N. Balakrishnan, and D. S. Zrnic, "Error structure of multiparameter radar and surface measurements of rainfall. Part III: Specific differential phase," J. Atmos. Oceanic Technol., vol. 7, no. 5, pp. 621-629, 1990.

[18] C. W. Ulbrich, "Natural variations in the analytical form of raindrop size distribution," J. Climate Appl. Meteorol., vol. 22, pp. 1764-1775, 1983.

[19] P. C. Waterman, Matrix formulation of electromagnetic scattering, Proc. IEEE, vol. 53, Aug. 1965.

[20] _ "Scattering by dielectric obstacles," Alta Frequenza, vol. 38 (special), pp. 348-352, 1969.

[21] V. N. Bringi and T. A. Seliga, "Scattering from axisymmetric dielectrics or perfect conductors imbedded in an axisymmetric dielectric," IEEE Trans. Antennas Propagat., succinct paper, vol. 25, pp. 575-580, 1977.

[22] J. Vivekanandan, W. M. Adams, and V. N. Bringi, "Rigorous approach to polarimetric radar modeling of hydrometeor orientation distributions," J. Appl. Meteorol., vol. 30, no. 8, pp. 1053-1063, Aug 1991.

[23] D. Zrnic, N. Balakrishnan, C. Ziegler, V. N. Bringi, K. Aydin, and T. Matejka, "Polarimetric signatures in the stratiform region of a mesoscale convective system," J. Appl. Meteorol., vol. 32, no. 4, pp. 678-693, Apr 1993.

[24] J. Goddard, P. Davies, and P. Hardaker, "Radar and multifrequency Olympus beacon measurements in heavy precipitation," IEE Conf. Publication, no. 370, 1993.

[25] A. Hendry, Y. M. M. Antar, J. J. Schlesak, and R. L. Olsen, "Melting layer attenuation at $28.6 \mathrm{GHz}$ from simultaneous COMSTAR beacon and polarization diversity radar data," Electron. Lett., vol. 17, no. 5, pp. 190-191, 1981. 
[26] _ "Melting layer attenuation at $28.6 \mathrm{GHz}$ from simultaneous COMSTAR beacon and $16.5 \mathrm{GHz}$ polarization diversity radar observations," IEE Conf. Publication no. 195: Second Intl. Conf. Antennas and Propagation, New York, 1981, pp. 12-16.

[27] B. C. Blevis, "Losses due to rain on radomes and antenna reflecting surfaces," IEEE Trans. Antennas Propagat., vol. AP13, pp. 175-176, Jan 1965.

[28] J. Beaver and V. N. Bringi, "Ka-band propagation studies using the ACTS propagation terminal and the CSU-CHILL multiparameter radar," paper presented at ACTS Propagations Studies Workshop (APSW IX), Virginia, Nov. 1996.

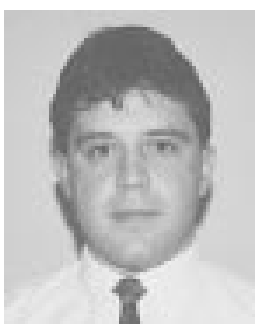

John D. Beaver received the B.S. degree in electrical engineering from Ohio University, Athens, in 1988, the M.S. degree in electrical engineering from Ohio State University, Columbus, in 1992, and the Ph.D. degree in electrical engineering from Colorado State University, Fort Collins, in 1996.

$\mathrm{He}$ is presently with Colorado State University in the CSU-CHILL remote sensing laboratory. His research interests include application of polarimetric radar techniques for attenuation prediction, modeling of cloud microphysics, and the study of propagation effects. His research experience also includes electromagnetic wave propagation and scattering theory.

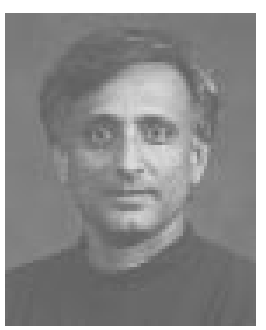

V. N. Bringi received the B.Tech.(Hons.) degree in electrical engineering from the Indian Institute of Technology, Bombay, India, in 1971, and the $\mathrm{Ph} . \mathrm{D}$. degree in electrical engineering from Ohio State University, Columbus, in 1976.

He joined Colorado State University, Fort Collins, in 1981 as an Associate Professor and became full Professor in 1987. His research interests are in the application of radar polarimetry to meteorology. He is Co-Principal Investigator for the CSU-CHILL national radar facility operated by Colorado State University under a five-year cooperative agreement with the National Science Foundation.

Dr. Bringi has served on the American Meteorological Society's Committee on Radar Meteorology. He is currently an Associate Editor of the Journal of Atmospheric and Oceanic Technology. 